\title{
Commutators, paraproducts and BMO in non-homogeneous martingale settings
}

\author{
Sergei Treil
}

\begin{abstract}
In this paper we investigate the relations between (martingale) BMO spaces, paraproducts and commutators in non-homogeneous martingale settings. Some new, and one might add unexpected, results are obtained. Some alternative proof of known results are also presented.
\end{abstract}

\section{Contents}

1 Introduction and main objects . . . . . . . . . . . . . . . . . . . 1326

1.1 Lattices, expectations and martingale differences . . . . . . . . . . . 1328

1.2 Martingale difference decomposition of $L^{p}$ spaces . . . . . . . . . . 1330

1.3 Martingale Hardy spaces . . . . . . . . . . . . . . . . . . . . . . . . 1331

1.4 Martingale transforms and martingale multipliers . . . . . . . . . . 1333

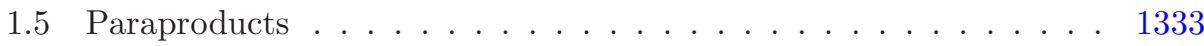

2 Triebel-Lizorkin type spaces . . . . . . . . . . . . . . . . . . . . . . . . . . . . . . . . . . . . . . . . . . .

2.1 Triebel-Lizorkin type spaces $\mathbf{g}_{p}^{q}(\mathcal{L}) \ldots \ldots \ldots \ldots$. . . . . . . 1337

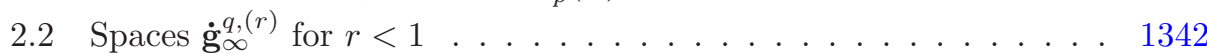

2.3 Embedding theorem for $\mathbf{g}_{p}^{q} \ldots \ldots \ldots \ldots \ldots \ldots$. . . . . . . . . . . . . . . . . . . . . . . 1344

$3 \mathcal{H}_{q}^{p}$ and $\mathcal{B M O}_{q}$ spaces . . . . . . . . . . . . . . . . . . . . 1347

$3.1 \mathcal{H}_{q}^{p}$ spaces . . . . . . . . . . . . . . . . . 1347

3.2 BMO spaces and $H^{1}$-BMO duality . . . . . . . . . . . . . . . . . . 1348

$3.3 \mathcal{B M O}_{q}$ as function spaces . . . . . . . . . . . . . . . . 1350

$4 \quad L^{p}$ bounds of paraproducts . . . . . . . . . . . . . . 1351

4.1 Martingale differences do not form a strong unconditional basis in $H^{p}$ in the non-homogeneous case . . . . . . . . . . . . . . . . . . 1351

4.2 "Paraproduct" version of the embedding theorem . . . . . . . . . 1357

4.3 Bounds for paraproducts . . . . . . . . . . . . . . 1361

5 Boundedness of commutators in $L^{p} \ldots \ldots \ldots$. . . . . . . . . . . . . . 1364

5.1 Sufficiency . . . . . . . . . . . . . . . . . 1364

Mathematics Subject Classification (2010): Primary 42B30; Secondary 42B25, 42C15.

Keywords: Paraproducts, commutators, BMO. 
$5.2 \quad$ Necessity . . . . . . . . . . . . . . . . . 1365

5.3 Relaxing the sufficient condition . . . . . . . . . . . . . . 1369

5.4 Some examples and counterexamples . . . . . . . . . . . . . 1370

\section{Notation}

$\mathbb{X}:$ real line $\mathbb{R}$ or its subinterval.

$\mathcal{L}$ : lattice of intervals in $\mathbb{X}$.

$\mathbf{1}_{I}$ : characteristic function of the set $I$.

$\langle f\rangle_{I}$ : average value of the function $f$ on $I$,

$$
\langle f\rangle_{I}=f_{I} f(x) d x:=|I|^{-1} \int_{I} f(x) d x .
$$

$\mathbb{E}_{I}$ : averaging operator, $\mathbb{E}_{I} f:=\langle f\rangle_{I} \mathbf{1}_{I}$.

$\Delta_{I}$ : martingale difference operator, $\Delta_{I}=\left(\sum_{J \in \operatorname{child}(I)} \mathbb{E}_{J}\right)-\mathbb{E}_{I} ;$ here $\operatorname{child}(I)$ denotes the collection of the "children" of $I$.

\section{Introduction and main objects}

This paper was started in an attempt to understand the relations between (martingale) commutators, paraproducts and space BMO. The initial hope was to cover both the one-parameter and multi-parameter cases, but it became clear at an early stage that in the general, non-homogeneous case, even the one-parameter situation is far from well understood.

While the results about $H^{1}$-BMO duality for general martingales are well known and can be considered classical, paraproducts and commutators have mostly been studied for regular $r$-adic martingales.

In this paper several new, and one might add unexpected, results are obtained for the non-homogeneous situation. Let me list some of them here; for the definitions and exact statements the reader should see the sections that follow.

- Despite what one might expect, the condition $b \in$ BMO is not necessary (although it is, of course, sufficient) for the boundedness of the paraproduct $\pi_{b}$ in $L^{p}$. This means, in particular, that unlike the homogeneous case it is impossible to characterize $b \in \mathrm{BMO}$ via boundedness of commutators of the multiplication operator $M_{b}$ and martingale multipliers.

The condition $b \in$ BMO however is necessary and sufficient for the boundedness of the so-called extended paraproduct $\pi_{b}^{(*)}$.

- The necessary and sufficient condition for the $L^{p}$ boundedness of the paraproduct is, as one might expect, that it is enough to check the boundedness on the characteristic functions of intervals. This statement is well known and now almost trivial for $p=2$; the result for $p \neq 2$ is new and its proof is rather complicated. 
Note that this condition depends on $p$, unlike the condition $b \in \mathrm{BMO}$, which guarantees the boundedness of $\pi_{b}^{(*)}$ in all $L^{p}, p \in(1, \infty)$.

- The condition $b \in \mathrm{BMO}$ is, as one might expect, sufficient for the $L^{p}$ boundedness $(p \in(1, \infty))$ of the commutator $\left[M_{b}, T\right]=M_{b} T-T M_{b}$ of the multiplication operator $M_{b}$ and a bounded martingale transform $T$. This condition (up to some technical details) is also necessary for the boundedness of the commutator, provided that the martingale transform $T$ satisfies some "mixing property".

This result generalizes the classical result of S. Janson [8], which gives the description of BMO via commutators in the case of regular $r$-adic martingales. The "mixing properties" that the martingale transform should satisfy generalize (and in the case of a regular $r$-adic lattice coincide with) the notion of the non-degenerate martingale transform, considered in [8].

The "mixing condition" introduced in this paper is necessarily more complicated than the non-degeneracy condition in [8]. This is mainly due to the fact that it includes a condition that was "hidden" (trivially satisfied) in the homogeneous case. An example, demonstrating that this "hidden" condition is essential is presented in the paper.

- It is shown in this paper that in the general non-homogeneous case the martingale difference spaces $D_{I}=\Delta_{I} L^{p}$ do not constitute a so-called strong unconditional basis in $L^{p}, p \neq 2$ (more precisely, in the martingale Hardy space $H^{p}$, which is, in general for $p \in(1, \infty)$ a subspace of $L^{p}$ with an equivalent metric). Essentially this means that it is impossible to define an equivalent norm in $H^{p}$ using only the norms of the martingale differences $\left\|\Delta_{I} f\right\|_{p}$.

An equivalent statement is that, unlike the case $p=2$, for each $p \neq 2$ one can construct a martingale transform (essentially a block diagonal operator, see the definition in the subsections that follow) with blocks $T_{I}$ uniformly bounded in $L^{p}$, which is not bounded in $L^{p}$.

A few words about general setup used in the paper. We do not work here in the setting of martingale spaces, because we want to include the situation with infinite measure, such as the standard dyadic lattice in $\mathbb{R}^{n}$. While getting results in the case of infinite measure from the corresponding result in the martingale case (the finite measure case) is usually pretty easy, there are some delicate situations, when one has to be careful stating the result. (Of course, usually after the results are stated, they are quite easy to prove, but stating the results requires some care).

For example, while this is well known to specialists, it might be a surprise to a reader only casually acquainted with martingale Hardy spaces and BMO, that for the standard dyadic lattice in $\mathbb{R}$ (and in $\mathbb{R}^{n}$ ) one can find a function $b$ in dyadic BMO such that the martingale difference decomposition $\sum_{I \in \mathcal{D}} \Delta_{I} b$ diverges a.e. I haven't seen this mentioned anywhere in the literature, probably everyone has had to notice this fact for him or herself.

So, in this paper we work on the real line $\mathbb{R}$, and our $\sigma$-algebras are generated by disjoint intervals. While practically everything can be stated and proved in the 
setting of arbitrary measure space, we want to avoid non-essential technical details and concentrate on the main ideas. For example, at some point we will be using the Fefferman-Stein maximal theorem, which is stated and proved for $\mathbb{R}^{n}$ but not for an arbitrary measure space.

The setting of the real line covers the example of principal interest: the case of $\mathbb{R}^{n}$ with the standard dyadic lattice and with an arbitrary Radon measure $\mu$, where the averages are taken with respect to $\mu$. Such a situation is typical in non-homogeneous harmonic analysis, see for example [10], [13] and [12].

\subsection{Lattices, expectations and martingale differences}

Let $\mathbb{X}$ be either the real line $\mathbb{R}$ or its subinterval (finite or infinite)0. A lattice $\mathcal{L}$ is a collection of nontrivial finite (bounded) intervals of $\mathbb{X}$ (say, for definiteness, of the form $[a, b))$ with the following properties:

(i) $\mathcal{L}$ is a union of generations $\mathcal{L}_{k}, k \in \mathbb{Z}$, where each generation is a collection of disjoint intervals, covering $\mathbb{X}$.

(ii) For each $k \in \mathbb{Z}$, the covering $\mathcal{L}_{k+1}$ is a finite refinement of the covering $\mathcal{L}_{k}$, i.e., each interval $I \in \mathcal{L}_{k}$ is a finite union of disjoint intervals $J \in \mathcal{L}_{k+1}$. We allow the situation where there is only one such interval $J$ (i.e., $J=I$ ); this means that $I \in \mathcal{L}_{k}$ also belongs to the generation $\mathcal{L}_{k+1}$.

(iii) $\bigcap_{k \in \mathbb{Z}} \mathcal{L}_{k}=\varnothing$, i.e., no interval $I$ belongs to all generations $\mathcal{L}_{k}$.

Remark. We allow situations where $I \in \mathcal{L}_{k}$ for all $k \geq N$ or for all $k<N$ for some $N \in \mathbb{Z}$, but the case when $I \in \mathcal{L}_{k}$ for all $k \in \mathbb{Z}$ is forbidden, because in the latter case nothing interesting happens on the interval $I$, as it does not interact with the rest of the space.

Example. The main example we have in mind is the following one. Consider the space $\mathbb{R}^{d}$ with a Radon measure $\mu$ and the standard dyadic lattice. Let us represent cubes $Q_{k}=Q_{k}^{1}=\left[0,2^{k}\right)^{d}$ by the intervals $I_{k}^{1}=\left[0, \mu\left(Q_{k}\right)\right) \subset \mathbb{R}$. For each cube $Q_{k}^{1}$ we pick some ordering of its children (dyadic subcubes of $Q_{k}^{1}$ with side $2^{k-1}$ ) with $Q_{k-1}^{1}=\left[0,2^{k-1}\right)^{d}$ being the first, and split $I_{k}^{1}$ into a disjoint union of intervals $I_{k-1}^{j}$, of the form $[a, b),\left|I_{k-1}^{j}\right|=\mu\left(Q_{k-1}^{j}\right)$, with the ordering of the intervals $I_{k-1}^{j}$ given by the ordering of $Q_{k-1}^{j}$.

Then we the order children of $Q_{k}^{j}, j \neq 1$, and represent them as subintervals of $I_{k}^{j}$, then order their children, and so on.

We have represented the standard dyadic lattice in the first "octant" $[0, \infty)^{d}$ of $\mathbb{R}^{d}$ with the measure $\mu$ by our lattice (with $\mathbb{X}=\left[0, \mu\left([0, \infty)^{d}\right)\right.$ ), so the measure of each dyadic cube equals the length of the corresponding interval. Note that the dyadic cubes $Q, \mu(Q)=0$, are ignored, as the corresponding intervals are empty sets.

If the measure $\mu$ is finite, we can represent the dyadic lattice in all of $\mathbb{R}^{d}$ as our lattice: in general, we can only put 2 "octants" on the line, but the dyadic lattice on the whole space can be represented as a finite disjoint union of our lattices. 
1.1.1. More definitions. For an interval $I \in \mathcal{L}$, let $\operatorname{rk}(I)$ be the rank of the interval $I$, i.e., the largest number $k$ such that $\mathcal{L}_{k} \ni I$. If no such number exists, i.e., if $I \in \mathcal{L}_{k}$ for all $k \geq N$, we say that $\operatorname{rk}(I)=\infty$.

For an interval $I \in \mathcal{L}, \operatorname{rk}(I)=k<\infty$, a child of $I$ is an interval $J \in \mathcal{L}_{k+1}$ such that $J \subset I$ (note that in this case an interval always has more than one child). If $\operatorname{rk}(I)=\infty$ we consider $I$ to be its own (and only) child. The collection of all children of an interval $I$ is denoted by child $(I)$.

We say that a lattice $\mathcal{L}$ is homogeneous if there exists $K<\infty$ such that $|I| /|J| \leq K$ for all $I \in \mathcal{L}, J \in \operatorname{child}(I)$.

Most of the results of this paper are well known for homogeneous lattices; the non-homogeneous case is the interesting one here.

1.1.2. Conditional expectations and martingale differences. For an interval $I \in \mathcal{L}$, let $\mathbb{E}_{I}$ be the averaging operator,

$$
\mathbb{E}_{I} f:=\left(|I|^{-1} \int_{I} f(x) d x\right) \mathbf{1}_{I}=:\langle f\rangle_{I} \mathbf{1}_{I},
$$

and let $\mathbb{E}_{k}$ be the "conditional expectation",

$$
\mathbb{E}_{k} f=\sum_{I \in \mathcal{L}_{k}} \mathbb{E}_{I} f
$$

Consider the martingale differences $\Delta_{I}$ and $\Delta_{k}$ :

$$
\Delta_{I}=\left(\sum_{J \in \operatorname{child}(I)} \mathbb{E}_{J}\right)-\mathbb{E}_{I}, \quad \Delta_{k}=\mathbb{E}_{k}-\mathbb{E}_{k-1}=\sum_{I \in \mathcal{L}: \operatorname{rk}(I)=k-1} \Delta_{I}
$$

(note that we cannot write $\Delta_{k}=\sum_{I \in \mathcal{L}_{k-1}} \Delta_{I}$ here).

Let $\mathfrak{A}_{k}$ be the $\sigma$-algebra generated by $\mathcal{L}_{k}$ (i.e., countable unions of intervals in $\left.\mathcal{L}_{k}\right)$. Let $\mathfrak{A}_{\infty}$ be the smallest $\sigma$-algebra containing all $\mathfrak{A}_{k}, k \in \mathbb{Z}$, and let $\mathfrak{A}_{-\infty}$ be the largest $\sigma$-algebra contained in all $\mathfrak{A}_{k}, \mathfrak{A}_{-\infty}=\cap_{k \in \mathbb{Z}} \mathfrak{A}_{k}$.

The structure of the $\sigma$-algebras $\mathfrak{A}_{\infty}$ and $\mathfrak{A}_{-\infty}$ is easy to understand. Thus, $\mathfrak{A}_{-\infty}$ is the $\sigma$-algebra generated by all the intervals $I$ of form

$$
I=\bigcup_{k \in \mathbb{Z}} I_{k}, \quad \text { where } I_{k} \in \mathcal{L}_{k}, I_{k} \subset I_{k-1} .
$$

Note that $\mathbb{X}$ is a disjoint union of such intervals $I$ and at most countably many points (we might need to add left endpoints to the intervals $I$, if they happen to be open intervals). It is possible that there is only one such $I, I=\mathbb{X}$, in which case the $\sigma$-algebra $\mathfrak{A}_{-\infty}$ is trivial.

Let us denote the collection of the intervals $I$ of form (1.1) by $\mathfrak{A}_{-\infty}^{0}$. Define

$$
\mathfrak{A}_{-\infty}^{0, \text { fin }}:=\left\{I \in \mathfrak{A}_{-\infty}^{0}:|I|<\infty\right\} ;
$$

here "fin" is included in the notation to remind that the set consists of intervals of finite measure.

For example, in the case of the standard dyadic lattice in $\mathbb{R}$, we have that $\mathfrak{A}_{-\infty}^{0}=\{[0, \infty),(-\infty, 0)\}$ and so $\mathfrak{A}_{-\infty}^{0, \text { fin }}=\varnothing$. 
Instead of describing $\mathfrak{A}_{\infty}$, let us describe the corresponding measurable functions. Namely, a function $f$ is $\mathfrak{A}_{\infty}$-measurable if it is Borel measurable and it is constant on intervals

$$
I=\bigcap_{k \in \mathbb{Z}} I_{k}, \quad \text { where } \quad I_{k} \in \mathcal{L}_{k}, I_{k} \subset I_{k-1} .
$$

Clearly, such intervals $I$ do not intersect, so there can only be countably many of them. Note, that if we assume that, for every $x \in \mathbb{X}$,

$$
\lim _{k \rightarrow+\infty}\left|I_{k}(x)\right|=0
$$

where $I_{k}(x)$ is the unique interval in $\mathcal{L}_{k}$ containing $x$, then $\mathfrak{A}_{\infty}$ is the Borel $\sigma$-algebra.

\subsection{Martingale difference decomposition of $L^{p}$ spaces}

In this paper we always assume that all functions are $\mathfrak{A}_{\infty}$-measurable.

One can easily see that

$$
\sum_{\substack{I \in \mathcal{L} \\ m \leq \operatorname{rk}(I)<n}} \Delta_{I}=\sum_{m<k \leq n} \Delta_{k}=\mathbb{E}_{n}-\mathbb{E}_{m} .
$$

Note that, for any $f \in L^{p}$ (as mentioned above, we assume here that all the functions are $\mathfrak{A}_{\infty}$-measurable),

$$
\mathbb{E}_{n} f \rightarrow f \quad \text { as } n \rightarrow+\infty,
$$

where the convergence is a.e. (for $p \in[1, \infty]$ ), and in the $L^{p}$ norm for $p \in[1, \infty)$.

To compute the limit $\mathbb{E}_{m} f$ as $m \rightarrow-\infty$, we notice that for a bounded compactly supported $f$ we can estimate $\left|\mathbb{E}_{I} f\right| \leq C /|I|$, so if $\left|I_{n}\right| \rightarrow \infty$ as $n \rightarrow \infty$, then for such functions and for $p \in(1, \infty]$

$$
\lim _{n \rightarrow \infty}\left\|\mathbb{E}_{I_{n}} f\right\|_{p}=0 \text {. }
$$

Since bounded compactly supported functions are dense in $L^{p}, p \in[1, \infty)$, and the operators $\mathbb{E}_{n}$ are contractions in $L^{p}$, we get, by applying the $\varepsilon / 3$ Theorem, that for $f \in L^{p}, p<\infty$,

$$
\mathbb{E}_{-n} f \rightarrow \sum_{I \in \mathfrak{A}_{-\infty}^{0, \text { fin }}} \mathbb{E}_{I} f \quad \text { as } n \rightarrow \infty
$$

where the convergence is in $L^{p}$ for $p \in(1, \infty)$ and in a weaker sense (say $L^{1}$ convergence on compacts) for $p=1$.

Therefore any function $f \in L^{p}, p \in(1, \infty)$ can be represented as an $L^{p}$ convergent series

$$
f=\sum_{I \in \mathcal{L}} \Delta_{I} f+\sum_{I \in \mathfrak{A}_{-\infty}^{0, \text { fin }}} \mathbb{E}_{I} f=\sum_{k \in \mathbb{Z}} \Delta_{k} f+\sum_{I \in \mathfrak{A}_{-\infty}^{0, \text { fin }}} \mathbb{E}_{I} f=\sum_{k \in \mathbb{Z}} \Delta_{k} f+\Delta_{-\infty} f
$$

we use the notation $\Delta_{-\infty}=\mathbb{E}_{-\infty}:=\sum_{I \in \mathfrak{A}_{-\infty}^{0, \text { fin }}} \mathbb{E}_{I}$ here. 
We have shown the convergence of the partial sums $\sum_{m}^{n}$, but in fact the convergence of the series is unconditional (independent of the ordering).

\subsection{Martingale Hardy spaces}

Everything in this subsection is well known. We present it only for the convenience of the reader.

Let us recall the classical result of D. Burkholder, which in our notation can be stated as follows.

Theorem 1.1 (D. Burkholder). Let $f$ and $g$ be two locally integrable functions on $\mathbb{X}$ such that, a.e. on $\mathbb{X}$,

$$
\left|\Delta_{I} f\right| \leq\left|\Delta_{I} g\right| \quad \forall I \in \mathcal{L}, \quad \text { and } \quad\left|\mathbb{E}_{I} f\right| \leq\left|\mathbb{E}_{I} g\right| \quad \forall I \in \mathfrak{A}_{-\infty}^{0, \text { fin }} .
$$

Then

$$
\|f\|_{p} \leq\left(p^{*}-1\right)\|g\|_{p},
$$

where $p^{*}=\max \left\{p, p^{\prime}\right\}, 1 / p+1 / p^{\prime}=1$.

In [2] this theorem was proved for arbitrary discrete time martingales, which immediately gives the above theorem in the special case where $|\mathbb{X}|=1$ an $\mathfrak{A}_{k}=\{\mathbb{X}\}$ for $k \leq 0$. The general statement can be obtained from this special case by easy and standard reasoning, which we skip.

Burkholder's theorem implies that, for $\left|\alpha_{k}\right|=1$,

$$
\frac{1}{C}\|f\|_{p} \leq\left\|\sum_{k \in \mathbb{Z} \cup\{-\infty\}} \alpha_{k} \Delta_{k} f\right\|_{p} \leq C\|f\|_{p},
$$

where $C=p^{*}-1$

Taking for the $\alpha_{k}$ independent Bernoulli random variables taking values \pm 1 with probability $1 / 2$, and taking expectations one gets

$$
\frac{1}{C^{p}}\|f\|_{p}^{p} \leq \int_{\Omega} \int_{\mathbb{X}}\left|\sum_{k \in \mathbb{Z} \cup\{-\infty\}} \alpha_{k}(\omega) \Delta_{k} f(x)\right|^{p} d x d P(\omega) \leq C^{p}\|f\|_{p} .
$$

Changing the order of integration, and noticing that, by the Khinchine inequality for any sequence of $x_{k} \in \mathbb{C}$, the averages

$$
\left(\int_{\Omega}\left|\sum_{k} \alpha_{k}(\omega) x_{k}\right|^{p} d P(\omega)\right)^{1 / p}
$$

and

$$
\left(\int_{\Omega}\left|\sum_{k} \alpha_{k}(\omega) x_{k}\right|^{2} d P(\omega)\right)^{1 / 2}=\left(\sum_{k}\left|x_{k}\right|^{2}\right)^{1 / 2}
$$

are equivalent with constants depending only on $p$, we can see that the quantity $\|\widetilde{S} f\|_{p}$, where $\widetilde{S} f$ is the so-called extended square function,

$$
\widetilde{S} f(x)=\left(\sum_{k \in \mathbb{Z} \cup\{-\infty\}}\left|\Delta_{k} f(x)\right|^{2}\right)^{1 / 2},
$$


defines an equivalent norm in $L^{p}$ (recall that we assume that all functions are $\mathfrak{A}_{\infty}$ measurable).

In particular, this implies that for $f \in L^{p}$ the sum in (1.4) converges unconditionally (independently of the ordering) in $L^{p}$. Note that if for a formal sum $f$ of form (1.4) we have $\widetilde{S} f \in L^{p}$, then the series converges unconditionally in $L^{p}$, so $L^{p}, p \in(1, \infty)$, is isomorphic to the set of formal series (1.4) with $\widetilde{S} f \in L^{p}$.

Let us also introduce the classical square function $S$, where we do not add the term $\left|\Delta_{-\infty} f\right|^{2}$,

$$
S f(x)=\left(\sum_{k \in \mathbb{Z}}\left|\Delta_{k} f(x)\right|^{2}\right)^{1 / 2} .
$$

The situation for $p=1$ is more interesting. Recall the classical result of Burges Davis [4] comparing the maximal function with the square function. Let us recall that the maximal function $M=M_{\mathcal{L}}$ is defined by

$$
M f(x):=\sup _{I \in \mathcal{L}: x \in I}\left|\mathbb{E}_{I} f\right|=\sup _{k \in \mathbb{Z}}\left|\mathbb{E}_{k} f(x)\right|
$$

Theorem 1.2 (B. Davis, 1970). Let $M=M_{\mathcal{L}}$ be the maximal function defined above, and let $\widetilde{S}(f)$ be the extended square function defined by (1.5). Then

$$
\frac{1}{C}\|M f\|_{1} \leq\|\widetilde{S} f\|_{1} \leq C\|M f\|_{1},
$$

where $C$ is an absolute constant.

Remark. The theorem in [4] was proved for general discrete time martingales, and in our case it can be applied directly to the situation where $|\mathbb{X}|=1, \mathfrak{A}_{k}=\{\mathbb{X}\}$ for $k \leq 0$, and $\mathbb{E}_{\mathbb{X}} f=0$. However, the general case can be easily obtained from this by standard reasoning, which we omit here.

Note that by the Lebesgue differentiation theorem, $\|f\|_{1} \leq\|M f\|_{1}$. Therefore, if $\left\|S f_{n}\right\|_{1} \rightarrow 0$, then $\left\|f_{n}\right\|_{1} \rightarrow 0$, so if $S f \in L^{1}$, then the martingale difference decomposition (1.4) converges unconditionally in $L^{1}$.

Definition. The martingale extended Hardy space $\widetilde{H}^{1}$ is the set of all functions $f \in L^{1}$ such that $\widetilde{S} f \in L^{1}$ (equivalently, $M f \in L^{1}$ ), equipped with the norm $\|f\|_{\widetilde{H}^{1}}=\|\widetilde{S} f\|_{1}$.

The Hardy space $H^{1}$ consists of all the functions in $\widetilde{H}^{1}$ such that $\mathbb{E}_{I} f=0$ for all $I \in \mathfrak{A}_{-\infty}^{0, \text { fin }}$ (with the norm given by $\|S f\|_{1}$ ). Note that $\|M f\|_{1}$ also gives an equivalent norm on $H^{1}$.

Remark. For $p \in(1, \infty)$ the extended martingale Hardy space $\widetilde{H}^{p}$ is also defined as the space of all locally integrable functions $f$ such that $\widetilde{S} f \in L^{p}$, with the norm $\|f\|_{\widetilde{H}^{p}}=\|S f\|_{p}$. While, as we discussed above, for $p \in(1, \infty)$ the space $\widetilde{H}^{p}$ is isomorphic to $L^{p}$, we will use the notation $\widetilde{H}^{p}$ as well (for example, to emphasize that we are using a different norm).

Finally, the spaces $H^{p}$ are defined as the subspaces of $\widetilde{H}^{p}$ comprising functions $f$ such that $\mathbb{E}_{I} f=0$ for all $I \in \mathfrak{A}_{-\infty}^{0, \text { fin }}$. 


\subsection{Martingale transforms and martingale multipliers}

Let $D_{I}:=\Delta_{I} L^{2}$. A martingale transform is a linear transformation $T$

$$
T\left(\sum_{I \in \mathcal{L}} \Delta_{I} f\right)=\sum_{I \in \mathcal{L}} T_{I}\left(\Delta_{I} f\right)
$$

where each $T_{I}$ is a linear transformation acting on the (finite-dimensional) space $D_{I}$. We also assume that $T \mathbb{E}_{I} f=0$ for all $I \in \mathfrak{A}_{-\infty}^{0, \text { fin }}$.

Such operators are well defined for finite sums; for now we will not assume the boundedness of $T$.

If all the operators $T_{I}$ are multiples of the identity, the corresponding martingale transform is called a martingale multiplier.

\subsection{Paraproducts}

For a function $b$ let us consider the multiplication operator $M_{b}, M_{b} f=b f$. We do not assume here that $M_{b}$ is bounded in $L^{2}$ (i.e., that $b \in L^{\infty}$ ). For our purposes, it is enough to assume that $b \in L_{\text {loc }}^{1}$, so that $\left\langle M_{b} f, g\right\rangle$ is well defined for $f$ and $g$ with finite martingale decompositions, i.e., for finite sums

$$
f=\sum_{I \in \mathcal{L}} \Delta_{I} f+\sum_{I \in \mathfrak{A}_{-\infty}^{0, \text { fin }}} \mathbb{E}_{I} f, \quad g=\sum_{I \in \mathcal{L}} \Delta_{I} g+\sum_{I \in \mathfrak{A}_{-\infty}^{0, \text { fin }}} \mathbb{E}_{I} g
$$

1.5.1. The "infinite measure" case. Let us first consider the situation where $\mathfrak{A}_{-\infty}^{0, \text { fin }}=\varnothing$. In this case, as was discussed above, the space $L^{2}$ is decomposed as the orthogonal sum of subspaces $D_{I}, I \in \mathcal{L}$.

Consider the decomposition

$$
M_{b} f=\sum_{I \in \mathcal{L}} \sum_{J \in \mathcal{L}} \Delta_{I} M_{b} \Delta_{J} f
$$

of the operator $M_{b}$ in this orthogonal basis. This sum can be split into 3 parts: over $I \varsubsetneqq J, J \varsubsetneqq I$ and $I=J$, respectively.

The first sum is called the paraproduct and is denoted by $\pi_{b} f$; the corresponding operator $\pi_{b}$ is also called the paraproduct. Since, for $I \varsubsetneqq J$,

$$
\Delta_{I}\left(b \Delta_{J} f\right)=\left(\Delta_{I} b\right)\left(\Delta_{J} f\right),
$$

we can write

$$
\pi_{b} f=\sum_{I, J \in \mathcal{L}: I \varsubsetneqq J} \Delta_{I}\left(b \Delta_{J} f\right)=\sum_{I, J \in \mathcal{L}: I \varsubsetneqq J}\left(\Delta_{I} b\right)\left(\Delta_{J} f\right)=\sum_{I \in \mathcal{L}}\left(\Delta_{I} b\right)\left(\mathbb{E}_{I} f\right) ;
$$

the last equality follows from the fact that, for fixed $I \in \mathcal{L}$,

$$
\sum_{J \in \mathcal{L}: I \varsubsetneqq J}\left(\Delta_{J} f\right) \mathbf{1}_{I}=\mathbb{E}_{I} f
$$


The second sum (over $J \varsubsetneqq I$ ) is $\pi_{b}^{*} f$, where $\pi_{b}^{*}$ is the dual of $\pi_{b}$ with respect to the standard linear duality $\langle f, g\rangle=\int f g$. This can be seen easily from the fact that $\left\langle\mathbb{E}_{I} f, g\right\rangle=\left\langle f, \mathbb{E}_{I} g\right\rangle$ and so $\left\langle\Delta_{I} f, g\right\rangle=\left\langle f, \Delta_{I} g\right\rangle$.

The third sum (over $I=J$ ) is the "diagonal" term denoted by $\Lambda_{b} f$. It is easy to see that

$$
\Lambda_{b} f=\sum_{I \in \mathcal{L}} \Delta_{I}\left(b \Delta_{I} f\right) .
$$

This diagonal term commutes with all martingale multipliers, so it can be ignored when one studies commutators of $M_{b}$ with martingale multipliers.

In the situation when all intervals $I \in \mathcal{L}$ have at most 2 children, any martingale transform is a multiplier, so in this case it is enough to consider the decomposition of $M_{b}$ as

$$
M_{b}=\pi_{b}+\pi_{b}^{*}+\Lambda_{b}
$$

where we can ignore the term $\Lambda_{b}$ when studying commutators with martingale transforms.

In the general situation, we can only ignore a term that is a martingale multiplier, so a different decomposition is needed. To present this decomposition we need the following lemma, which gives us a formula for $\pi_{b}^{*}$.

Lemma 1.3. The (formal) dual $\pi_{b}^{*}$ of $\pi_{b}$ with respect to the standard linear duality is given by

$$
\pi_{b}^{*} f=\sum_{I \in \mathcal{L}} \mathbb{E}_{I}\left(\left(\Delta_{I} b\right)\left(\Delta_{I} f\right)\right)=\sum_{I \in \mathcal{L}} \mathbb{E}_{I}\left(\left(b-\mathbb{E}_{I} b\right)\left(\Delta_{I} f\right)\right)=\sum_{I \in \mathcal{L}} \mathbb{E}_{I}\left(b\left(\Delta_{I} f\right)\right) .
$$

The word "formal" here means that the equality $\left\langle\pi_{b} f, g\right\rangle=\left\langle f, \pi_{b}^{*} g\right\rangle$ holds for all finite sums $f=\sum_{I \in \mathcal{L}} \Delta_{I} f, g=\sum_{I \in \mathcal{L}} \Delta_{I} g$.

Proof. It is easy to see that $\left\langle\mathbb{E}_{I} f, g\right\rangle=\left\langle f, \mathbb{E}_{I} g\right\rangle$, and so $\left\langle\Delta_{I} f, g\right\rangle=\left\langle f, \Delta_{I} g\right\rangle$. Using these identities and the fact that $\left(\Delta_{I} b\right)\left(\mathbb{E}_{I} f\right)=\Delta_{I}\left(b \mathbb{E}_{I} f\right)$, we get

$$
\begin{aligned}
\left\langle\pi_{b} f, g\right\rangle & =\sum_{I \in \mathcal{L}}\left\langle\Delta_{I}\left(b \mathbb{E}_{I} f\right), g\right\rangle=\sum_{I \in \mathcal{L}}\left\langle b \mathbb{E}_{I} f, \Delta_{I} g\right\rangle \\
& =\sum_{I \in \mathcal{L}}\left\langle\mathbb{E}_{I} f, b \Delta_{I} g\right\rangle=\sum_{I \in \mathcal{L}}\left\langle f, \mathbb{E}_{I}\left(b \Delta_{I} g\right)\right\rangle .
\end{aligned}
$$

To complete the proof it remains to show that

$$
\mathbb{E}_{I}\left(b \Delta_{I} g\right)=\mathbb{E}_{I}\left(\left(b-\mathbb{E}_{I} b\right)\left(\Delta_{I} g\right)\right)=\mathbb{E}_{I}\left(\left(\Delta_{I} b\right)\left(\Delta_{I} g\right)\right),
$$

which we leave as an exercise for the reader.

To give an alternative (to (1.9)) decomposition of $M_{b}$ let us notice that

$$
\Delta_{I}\left(b \Delta_{I} f\right)=\Delta_{I}\left(\left(b-\mathbb{E}_{I} b\right) \Delta_{I} f\right)+\left(\mathbb{E}_{I} b\right) \Delta_{I} f=\Delta_{I}\left(\left(\Delta_{I} b\right) \Delta_{I} f\right)+\left(\mathbb{E}_{I} b\right) \Delta_{I} f .
$$


Therefore, we can decompose $\Lambda_{b}=\Lambda_{b}^{1}+\Lambda_{b}^{0}$, where

$$
\begin{aligned}
& \Lambda_{b}^{1} f=\sum_{I \in \mathcal{L}} \Delta_{I}\left[\left(\Delta_{I} b\right)\left(\Delta_{I} f\right)\right], \\
& \Lambda_{b}^{0} f=\sum_{I \in \mathcal{L}}\left(\mathbb{E}_{I} b\right)\left(\Delta_{I} f\right) .
\end{aligned}
$$

Note, that $\Lambda_{b}^{0}$ is a martingale multiplier, so it commutes with all martingale transforms.

Defining

$$
\pi_{b}^{(*)}:=\pi_{b}^{*}+\Lambda_{b}^{1}
$$

we can decompose the multiplication operator $M_{b}, M_{b} f:=b f$ as

$$
M_{b}=\pi_{b}+\pi_{b}^{(*)}+\Lambda_{b}^{0} .
$$

\section{Lemma 1.4.}

$$
\pi_{b}^{(*)} f=\sum_{I \in \mathcal{L}}\left(\Delta_{I} b\right)\left(\Delta_{I} f\right)
$$

Proof. Notice that

$$
\left(\Delta_{I} b\right)\left(\Delta_{I} f\right)=\mathbb{E}_{I}\left(\left(\Delta_{I} b\right)\left(\Delta_{I} f\right)\right)+\Delta_{I}\left(\left(\Delta_{I} b\right)\left(\Delta_{I} f\right)\right) .
$$

Taking the sum over all $I \in \mathcal{L}$ we get $\pi_{b}^{*} f+\Lambda_{b}^{1} f$ on the right side, which proves the lemma.

There is an alternative, probably a more natural way, to get the decomposition (1.13). Namely, let us consider the product $b f$, which can be written as

$$
\sum_{I, J \in \mathcal{L}}\left(\Delta_{I} b\right)\left(\Delta_{J} f\right)
$$

(let us not worry about convergence here and assume that the sums in the martingale difference decompositions of $f$ and $b$ are finite).

Let us split the above sum into 3 parts, over the sets $I \varsubsetneqq J, J \varsubsetneqq I$ and $I=J$ respectively.

The first sum gives us the paraproduct $\pi_{b} f$ :

$$
\sum_{I, J \in \mathcal{L}: I \varsubsetneqq J}\left(\Delta_{I} b\right)\left(\Delta_{J} f\right)=\sum_{I \in \mathcal{L}}\left(\Delta_{I} b\right)\left(\mathbb{E}_{I} f\right)=: \pi_{b} f ;
$$

see (1.8).

The second sum (over $J \varsubsetneqq I$ ) can be written as $\pi_{f} b$, so, using (1.8) with $f$ and $b$ interchanged and recalling the definition of $\Lambda_{b}^{0}$, see (1.11), we get

$$
\sum_{I, J \in \mathcal{L}: J \subsetneq I}\left(\Delta_{I} b\right)\left(\Delta_{J} f\right)=\sum_{J \in \mathcal{L}}\left(\mathbb{E}_{J} b\right)\left(\Delta_{J} f\right)=: \Lambda_{b}^{0} f .
$$


Finally, the last sum gives us (see (1.14))

$$
\sum_{I \in \mathcal{L}}\left(\Delta_{I} b\right)\left(\Delta_{I} f\right)=: \pi_{b}^{(*)} f
$$

Remark. Note, that if $\mathcal{L}$ is the standard dyadic lattice, then $\mathbb{E}_{I}\left(\left(\Delta_{I} b\right)\left(\Delta_{I} f\right)\right)=$ $\left.\left(\Delta_{I} b\right)\left(\Delta_{I} f\right)\right)$, so $\pi_{b}^{(*)}=\pi_{b}^{*}$. This fact was used, for example, in [1].

1.5.2. Paraproducts in the general case. Let us now consider the general case, where $\mathfrak{A}_{-\infty}^{0, \text { fin }} \neq \varnothing$. Consider the decompositions

$$
f=\sum_{I \in \mathcal{L}} \Delta_{I} f+\sum_{I \in \mathfrak{A}_{-\infty}^{0, \text { fin }}} \mathbb{E}_{I} f, \quad g=\sum_{I \in \mathcal{L}} \Delta_{I} g+\sum_{I \in \mathfrak{A}_{-\infty}^{0, \text { fin }}} \mathbb{E}_{I} g,
$$

and let us decompose $\langle b f, g\rangle$. For a fixed $I \in \mathcal{L}$

$$
\left(\sum_{J \in \mathcal{L}: J \supsetneqq I} \Delta_{J} f+\sum_{J \in \mathfrak{A}_{-\infty}^{0, \text { fin }}: J \supset I} \mathbb{E}_{J} f\right) \mathbf{1}_{I}=\mathbb{E}_{I} f
$$

(note that the second sum on the left-hand side has at most one term). Therefore,

$$
\left\langle b\left(\sum_{J \in \mathcal{L}: J \supsetneqq I} \Delta_{J} f+\sum_{J \in \mathfrak{A}_{-\infty}^{0, f i n}: J \supset I} \mathbb{E}_{J} f\right), \Delta_{I} g\right\rangle=\left\langle\left(\Delta_{I} b\right) \mathbb{E}_{I} f, \Delta_{I} g\right\rangle=\left\langle\pi_{b} f, \Delta_{I} g\right\rangle,
$$

where, as above,

$$
\pi_{b} f:=\sum_{I \in \mathcal{L}}\left(\mathbb{E}_{I} f\right)\left(\Delta_{I} b\right)
$$

Similarly,

$$
\left\langle b \Delta_{I} f,\left(\sum_{J \in \mathcal{L}: J \supsetneqq I} \Delta_{J} g+\sum_{J \in \mathfrak{A}_{-\infty}^{0, \text { fin }}: J \supset I} \mathbb{E}_{I} g\right)\right\rangle=\left\langle\Delta_{I} f,\left(\Delta_{I} b\right) \mathbb{E}_{J} g\right\rangle=\left\langle\Delta_{I} f, \pi_{b} g\right\rangle .
$$

As we discussed above,

$$
\sum_{I \in \mathcal{L}}\left\langle b \Delta_{I} f, \Delta_{I} g\right\rangle=\left\langle\Lambda_{b} f, g\right\rangle
$$

where

$$
\Lambda_{b} f:=\sum_{I \in \mathcal{L}} \Delta_{I}\left(b \Delta_{I} f\right)
$$

The only terms in $\langle f, g\rangle$ that we did not yet count are the terms with $I, J \in \mathfrak{A}_{-\infty}^{0 \text {,fin }}$, which give us the remainder

$$
\sum_{I \in \mathfrak{A}_{-\infty}^{0, \text { fin }}}\left\langle b \mathbb{E}_{I} f, \mathbb{E}_{I} g\right\rangle=\left\langle\sum_{I \in \mathfrak{A}_{-\infty}^{0, \text { fin }}}\left(\mathbb{E}_{I} b\right) \mathbb{E}_{I} f, \mathbb{E}_{I} g\right\rangle=:\left\langle R_{b} f, g\right\rangle .
$$


So, the multiplication operator $M_{b}$ can be decomposed as

$$
M_{b}=\pi_{b}^{*}+\Lambda_{b}+\pi_{b}+R_{b},
$$

where the paraproduct $\pi_{b}$ is defined by $(1.16), \pi_{b}^{*}$ is its adjoint, $\Lambda_{b}$ is defined by (1.17), and

$$
R_{b} f=\left(\mathbb{E}_{-\infty} b\right)\left(\mathbb{E}_{-\infty} f\right)=\sum_{I \in \mathfrak{A}_{-\infty}^{0, \text { fin }}}\left(\mathbb{E}_{I} b\right)\left(\mathbb{E}_{I} f\right) .
$$

Note that Lemma 1.3 remains true in the general case as well: the proof is exactly the same. Also, nothing changes in the decomposition $\Lambda_{b}=\Lambda_{b}^{0}+\Lambda_{b}^{1}$, because we can investigate this decomposition separately in each block $D_{I}$, and these blocks know nothing about $\mathfrak{A}_{-\infty}^{0, \text { fin }}$. Finally, the proof of Lemma 1.4 works in the general case without any changes.

Summarizing we can state the following proposition.

Proposition 1.5. The multiplication operator $M_{b}$ is represented (at least formally) as

$$
M_{b}-R_{b}=\pi_{b}^{(*)}+\Lambda_{b}^{0}+\pi_{b}=\pi_{b}^{*}+\Lambda_{b}+\pi_{b}=\pi_{b}^{*}+\Lambda_{b}^{0}+\Lambda_{b}^{1}+\pi_{b},
$$

where

$$
\begin{array}{ll}
\Lambda_{b} f=\sum_{I \in \mathcal{L}} \Delta_{I}\left(b \Delta_{I} f\right), & \Lambda_{b}=\Lambda_{b}^{0}+\Lambda_{b}^{1} \\
\Lambda_{b}^{0} f & =\sum_{I \in \mathcal{L}}\left(\mathbb{E}_{I} b\right)\left(\Delta_{I} f\right), \quad \Lambda_{b}^{1} f=\sum_{I \in \mathcal{L}} \Delta_{I}\left[\left(\Delta_{I} b\right)\left(\Delta_{I} f\right)\right] .
\end{array}
$$

\section{Triebel-Lizorkin type spaces}

This part is devoted to the investigation of the "coefficient space" of the spaces $H^{p}$. We are mostly interested in the spaces with $q=2$, but since the results for $q \neq 2$ are often obtained with little or no extra effort, we consider here the case of general $q$.

The notation $\dot{\mathbf{g}}_{p}^{q}$ is chosen by analogy with the notation $\dot{\mathbf{f}}_{p}^{\alpha, q}$ for Triebel-Lizorkin spaces, see for example [6]. Since we use a different scaling here, to avoid confusion we use different notation. Also, we do not use the smoothness parameter $\alpha$ (we do not need it in what follows, and frankly, it is not completely clear what is the correct smoothness in the general non-homogeneous case). For the standard dyadic lattices in $\mathbb{R}^{d}$ our spaces $\dot{\mathbf{g}}_{p}^{q}$ are isomorphic to $\dot{\mathbf{f}}_{p}^{0, q}$, with isomorphism given by rescaling of the entries.

\subsection{Triebel-Lizorkin type spaces $\dot{\mathrm{g}}_{p}^{q}(\mathcal{L})$}

Let $\mathcal{L}$ be a lattice. For $1 \leq p, q<\infty$ define the sequence spaces $\dot{\mathbf{g}}_{p}^{q}(\mathcal{L})$ to consist of sequences $s=\left\{s_{I}\right\}_{I \in \mathcal{L}}$ such that

$$
\|s\|_{\dot{\mathbf{g}}_{p}^{q}(\mathcal{L})}:=\left\|\left(\sum_{I \in \mathcal{L}}\left|s_{I}\right|^{q} \mathbf{1}_{I}\right)^{1 / q}\right\|_{L^{p}}<\infty .
$$


For $p=\infty$ the norm is defined using the BMO-like norm

$$
\|s\|_{\dot{\mathbf{g}}_{\infty}^{q}(\mathcal{L})}:=\sup _{J \in \mathcal{L}}\left(\frac{1}{|J|} \int_{J} \sum_{I \in \mathcal{L}, I \subset J}\left|s_{I}\right|^{q} \mathbf{1}_{I}\right)^{1 / q} .
$$

Formally, one can define the whole scale of spaces $\dot{\mathbf{g}}_{\infty}^{q,(r)}(\mathcal{L}), 1 \leq r<\infty$,

$$
\|s\|_{\dot{\mathbf{g}}_{\infty}^{q,(r)}(\mathcal{L})}:=\sup _{J \in \mathcal{L}}\left(\frac{1}{|J|} \int_{J}\left(\sum_{I \in \mathcal{L}, I \subset J}\left|s_{I}\right|^{q} \mathbf{1}_{I}\right)^{r / q}\right)^{1 / r}
$$

but it will be shown later that these norms are equivalent for $1 \leq r<\infty$.

To shorten the notation, we will omit $\mathcal{L}$ and use the notation $\dot{\mathrm{g}}_{p}^{q}$ instead of $\dot{\mathbf{g}}_{p}^{q}(\mathcal{L})$, when it is clear from the context what the lattice $\mathcal{L}$ is.

The spaces $\dot{\mathbf{g}}_{p}^{q}(\mathcal{L})$ can be naturally identified with subspaces of $L^{p}\left(\ell^{q}\right)\left(L^{p}\right.$ with values in $\ell^{q}$ ). Namely, for a sequence $s=\left\{s_{I}\right\}_{I \in \mathcal{L}}$, define functions

$$
f_{k}=\sum_{I \in \mathcal{L}: \mathrm{rk}(I)=k} s_{I} \mathbf{1}_{I}, \quad k \in \overline{\mathbb{Z}}:=\mathbb{Z} \cup\{\infty\},
$$

and let

$$
f(x, k)=f_{k}(x), \quad k \in \overline{\mathbb{Z}}, \quad x \in \mathbb{R} .
$$

Then clearly, for $1 \leq p, q<\infty$

$$
\|s\|_{\dot{\mathbf{g}}_{p}^{q}(\mathcal{L})}=\|f\|_{L^{p}\left(\ell^{q}\right)}:=\left(\int\|f(x, \cdot)\|_{\ell^{q}}^{p} d x\right)^{1 / p} .
$$

Thus, the space $\mathbf{g}_{p}^{q}(\mathcal{L}), 1 \leq p, q<\infty$ can be naturally identified with the subspace of $L^{p}\left(\ell^{q}\right)$ consisting of functions $f$ such that $f(\cdot, k)$ is constant on intervals $I \in \mathcal{L}, \operatorname{rk}(I)=k$, and such that $f(x, k)=0$ if there is no interval $I \in \mathcal{L}, \operatorname{rk}(I)=k$, containing $x$ (recall that $\operatorname{rk}(I)$ is the largest integer $k$ such that $I \in \mathcal{L}_{k}$, so the condition $I \in \mathcal{L}_{k}$ does not mean that $\operatorname{rk}(I)=k$ ).

We will routinely switch between the function and sequence representation of elements of $\dot{\mathbf{g}}_{P}^{q}$, so regarding $f \in \dot{\mathbf{g}}_{p}^{q}$ as a sequence $\left\{f_{I}\right\}_{I \in \mathcal{L}}$ or as the corresponding function $f(\cdot, \cdot) \in L^{p}\left(\ell^{q}\right)$.

We will also need the notion of the coordinate projection of $f \in \dot{\mathrm{g}}_{p}^{q}$. Namely, for $\mathcal{E} \subset \mathcal{L}$ define the coordinate projection $f_{\mathcal{E}}$ by

$$
f_{\mathcal{E}}=\left\{f_{I}\right\}_{I \in \mathcal{E}}
$$

(meaning that entries corresponding to $I \notin \mathcal{E}$ are 0 ). In the function representation this can be written as

$$
f_{\mathcal{E}}(\cdot, k)=f(\cdot, k) \cdot\left(\sum_{I \in \mathcal{E}: \operatorname{rk}(I)=k} \mathbf{1}_{I}\right), \quad k \in \mathbb{Z} \cup\{\infty\} .
$$

For $f \in L^{p}\left(\ell^{q}\right)$ define the vector Hardy-Littlewood maximal function $f^{*}$ :

$$
f^{*}(x, k)=\sup _{I \ni x} \frac{1}{|I|} \int_{I}|f(s, k)| d s .
$$

We will need the following well-known theorem: 
Theorem 2.1 (Fefferman-Stein, [5]). Let $f \in L^{p}\left(\ell^{q}\right), 1<p, q<\infty$. Then

$$
\left\|f^{*}\right\|_{L^{p}\left(\ell^{q}\right)} \leq C\|f\|_{L^{p}\left(\ell^{q}\right)},
$$

where $C$ depends only on $p$ and $q$.

The following fact is well known.

Proposition 2.2. For $1<p, q<\infty$, the dual space $\left(\dot{\mathbf{g}}_{p}^{q}(\mathcal{L})\right)^{*}$ is isomorphic to $\mathbf{\mathrm { g }}_{p^{\prime}}^{q^{\prime}}(\mathcal{L})$, where $1 / p+1 / p^{\prime}=1,1 / q+1 / q^{\prime}=1$, and the pairing is the standard one:

$$
\langle f, g\rangle=\int_{\mathbb{X}} \sum_{k} f(x, k) g(x, k) d x=\int_{\mathbb{X}} \sum_{I \in \mathcal{L}} f_{I} g_{I} \mathbf{1}_{I}=\sum_{I \in \mathcal{L}} f_{I} g_{I}|I| .
$$

Note that we only claim that the norm in $\mathbf{\mathbf { g }}_{p^{\prime}}^{q^{\prime}}(\mathcal{L})$ is equivalent to the norm in the dual space (except in the trivial case $p=q=2$, where the norms coincide).

For the sake of completeness we present the proof of this proposition.

Proof. Since $\left(L^{p}\left(\ell^{q}\right)\right)^{*}=L^{p^{\prime}}\left(\ell^{q^{\prime}}\right)$ (for $\left.1<p, q<\infty\right)$, any $g \in \dot{\mathbf{g}}_{p^{\prime}}^{q^{\prime}}(\mathcal{L})$ defines a bounded linear functional $L$ on $\dot{\mathbf{g}}_{p}^{q}(\mathcal{L})$, and $\|L\| \leq\|g\|_{\dot{\mathbf{g}}_{p^{\prime}}^{q^{\prime}}(\mathcal{L})}$.

On the other hand, if $L$ is a bounded linear functional on $\dot{\mathbf{g}}_{p}^{q}(\mathcal{L})$, it can be extended by the Hahn-Banach theorem to a bounded linear functional on $L^{p}\left(\ell^{q}\right)$, which can be represented by a function $\widetilde{g} \in L^{p^{\prime}}\left(\ell^{q^{\prime}}\right)$,

$$
L(f)=\int \sum_{k} f(x, k) \widetilde{g}(x, k) d x, \quad \forall f \in \mathbf{g}_{p}^{q}(\mathcal{L}) .
$$

Note that the functional $L$ does not change if we replace the function $\widetilde{g}$ by its "orthogonal" projection $g$ onto $\dot{\mathrm{g}}_{p^{\prime}}^{q^{\prime}}(\mathcal{L})$,

$$
g(x, k)= \begin{cases}|I|^{-1} \int_{I} \widetilde{g}(s, k) d s, & \text { if } \operatorname{rk}(I)=k, \text { and } x \in I, \\ 0 & \text { if } \nexists I \ni x, \operatorname{rk}(I)=k .\end{cases}
$$

Clearly $|g| \leq(\widetilde{g})^{*}$, so by the Fefferman-Stein maximal theorem (Theorem 2.1),

$$
\|g\|_{L^{p^{\prime}}\left(\ell^{q^{\prime}}\right)} \leq C\|\widetilde{g}\|_{L^{p^{\prime}}\left(\ell^{q^{\prime}}\right)} .
$$

The dual of $\dot{\mathbf{g}}_{1}^{q}$ is given by the following theorem.

Theorem 2.3. Let $1<q<\infty$. Then the spaces $\dot{\mathbf{g}}_{\infty}^{q,(r)}, 1 \leq r<\infty$ do not depend on $r$, and the corresponding norms are equivalent. Moreover, the dual space $\left(\mathbf{g}_{1}^{q}\right)^{*}$ is isomorphic to $\dot{\mathbf{g}}_{\infty}^{q^{\prime}}:=\dot{\mathbf{g}}_{\infty}^{q^{\prime},\left(q^{\prime}\right)} \cong \mathbf{g}_{\infty}^{q^{\prime},(r)}$; here again $1 / q+1 / q^{\prime}=1$ and the pairing is given by $(2.3)$.

The notion of maximality, given in the definition below, plays an important role in the proof of Theorem 2.3, as well as later in the paper. 


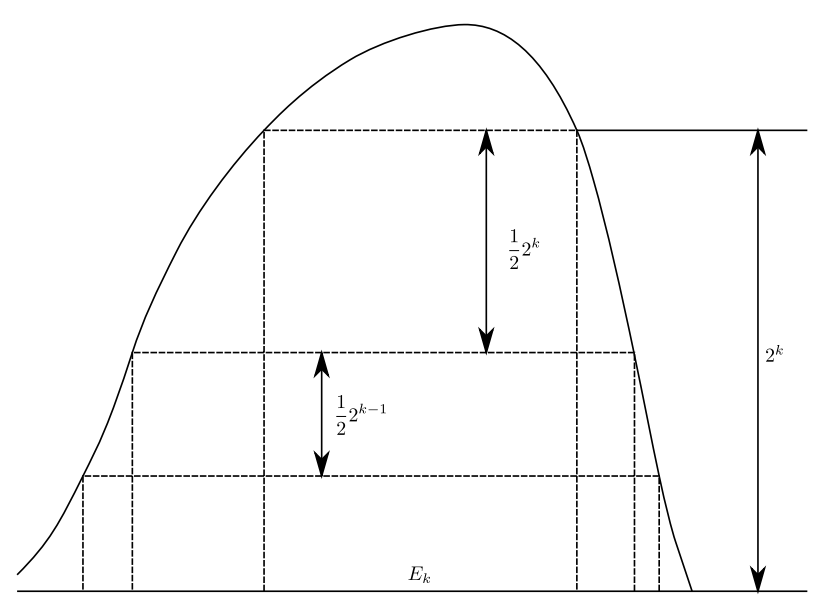

Figure 1. Level sets.

Definition. Let $\mathcal{E} \subset \mathcal{L}$. We say that $I \in \mathcal{E}$ is maximal for $\mathcal{E}$, or $\mathcal{E}$-maximal, if there is no $J \in \mathcal{E}$ such that $I \varsubsetneqq J$.

We will use the following simple lemma often.

Lemma 2.4. Let $\Phi: \mathbb{X} \rightarrow[0, \infty)$ and let $E_{k}:=\left\{x \in \mathbb{X}: \Phi(x)>2^{k}\right\}$. Then for $p>0$,

$$
\sum_{k \in \mathbb{Z}} 2^{k p}\left|E_{k}\right| \leq 2^{p} \int_{\mathbb{X}} \Phi(x)^{p} d x .
$$

Proof. See Figure 1.

Proof of Theorem 2.3. The general outline of the proof is as follows. We first show that

$$
|\langle f, g\rangle| \leq 4\|f\|_{\dot{\mathbf{g}}_{1}^{q}}\|g\|_{\dot{\mathbf{g}}_{\infty}^{q^{\prime},(1)}} \quad \forall f \in \dot{\mathbf{g}}_{1}^{q}, \forall g \in \dot{\mathbf{g}}_{\infty}^{q^{\prime},(1)}
$$

Testing a functional $\varphi \in\left(\dot{\mathbf{g}}_{1}^{q}\right)^{*}$ on sequences with one nonzero term, one can easily see that there exists a unique sequence $g=g_{\varphi}=\left\{g_{I}\right\}_{I \in \mathcal{L}}$ such that $\varphi(f)=$ $\langle f, g\rangle$ for all sequences $f$ with finitely many nonzero terms. We will show that, for all $r \in(1, \infty)$,

$$
\left\|g_{\varphi}\right\|_{\dot{\mathbf{g}}_{\infty}^{q^{\prime},(r)}} \leq C(r)\|\varphi\|
$$

It easily follows from the Hölder inequality that, for $r>1$,

$$
\|g\|_{\dot{\mathbf{g}}_{\infty}^{q^{\prime},(1)}} \leq\|g\|_{\dot{\mathbf{g}}_{\infty}^{q^{\prime},(r)}}
$$


The estimate $(2.5)$ means that $\|\varphi\| \leq 4\left\|g_{\varphi}\right\|_{\dot{\mathbf{g}}_{\infty}^{q^{\prime},(1)}}$. Combining this with (2.7) and (2.6) we get that

$$
\|\varphi\| \leq 4\left\|g_{\varphi}\right\|_{\dot{\mathbf{g}}_{\infty}^{q^{\prime},(1)}} \leq 4\left\|g_{\varphi}\right\|_{\dot{\mathbf{g}}_{\infty}^{q^{\prime},(r)}} \leq 4 C(r)\|\varphi\| .
$$

This proves the equivalence of the norms $\left\|g_{\varphi}\right\|_{\dot{\mathbf{g}}_{\infty}^{q^{\prime}},(r)}, r \in[1, \infty)$, and $\|\varphi\|$, and so the theorem.

Let us prove (2.5). It is sufficient to prove this inequality on a dense set of functions $f$ for which the corresponding sequence $\left\{s_{I}\right\}_{I \in \mathcal{L}}$ has finitely many nonzero terms.

Let $E_{k}:=\left\{x \in \mathbb{R}:\|f(x, \cdot)\|_{\ell_{q}}>2^{k}\right\}$, and let $\mathcal{E}_{k}:=\left\{I \in \mathcal{L}: I \subset E_{k}\right\}$. Note that $E_{k}$ is a finite disjoint union of $\mathcal{E}_{k}$-maximal intervals $I \in \mathcal{E}_{k}$.

Lemma 2.4 implies that

$$
\sum_{k \in \mathbb{Z}} 2^{k}\left|E_{k}\right| \leq 2 \int\|f(x, \cdot)\|_{\ell_{q}} d x .
$$

Since $\mathcal{L}$ is a disjoint union of the sets $\mathcal{E}_{k} \backslash \mathcal{E}_{k+1}$ we write

$$
f=\sum f_{\mathcal{E}_{k} \backslash \mathcal{E}_{k+1}}
$$

where $f_{\mathcal{E}}$ is the coordinate projection of $f$, see (2.1) and (2.2). Note that

$$
\left\|f_{\mathcal{E}_{k} \backslash \mathcal{E}_{k+1}}(x, \cdot)\right\|_{\ell^{q}} \leq 2^{k+1} \mathbf{1}_{E_{k}}(x) .
$$

Indeed, the estimate for $x \notin E_{k+1}$ is trivial. For $x \in E_{k+1}$, let $J$ be the $\mathcal{E}_{k+1}$-maximal interval containing $x$, and let $\widetilde{J}$ be the parent of $J$. Clearly $\widetilde{J} \notin \mathcal{E}_{k+1}$, so there exists a point $y \in \widetilde{J}, y \notin E_{k+1}$. Then we can write

$$
\begin{aligned}
\left\|f_{\mathcal{E}_{k} \backslash \mathcal{E}_{k+1}}(x, \cdot)\right\|_{\ell^{q}}^{q} & =\sum_{I \in \mathcal{E}_{k}: I \supsetneqq J}\left|f_{I}\right|^{q}=\sum_{I \in \mathcal{E}_{k}: I \supset \widetilde{J}}\left|f_{I}\right|^{q} \\
& \leq \sum_{I \in \mathcal{L}: I \ni y}\left|f_{I}\right|^{q}=\|f(y, \cdot)\|_{\ell^{q}}^{q} \leq 2^{(k+1) q}
\end{aligned}
$$

(the last inequality holds because $\left.y \notin E_{k+1}\right)$, and (2.9) is proved.

To finish the proof of (2.5) let us take $g \in \dot{\mathbf{g}}_{\infty}^{q^{\prime},(1)},\|g\|_{\dot{\mathbf{g}}_{\infty}^{q^{\prime},(1)}} \leq 1$, and estimate

$$
\begin{aligned}
|\langle f, g\rangle| & =\left|\int \sum_{I \in \mathcal{L}} f_{I} g_{I} \mathbf{1}_{I} d x\right| \leq \sum_{k \in \mathbb{Z}} \int_{I \in \mathcal{E}_{k} \backslash \mathcal{E}_{k+1}}\left|f_{I}\right|\left|g_{I}\right| \mathbf{1}_{I} d x \\
& \leq \sum_{k \in \overline{\mathbb{Z}}}\left\|f_{\mathcal{E}_{k} \backslash \mathcal{E}_{k+1}}\right\|_{L^{\infty}\left(\ell^{q}\right)}\left\|g_{\mathcal{E}_{k} \backslash \mathcal{E}_{k+1}}\right\|_{L^{1}\left(\ell^{q^{\prime}}\right)} \leq \sum_{k \in \mathbb{Z}} 2^{k+1}\left|E_{k}\right| \leq 4\|f\|_{L^{1}\left(\ell^{q}\right)} .
\end{aligned}
$$

Here the last inequality follows from (2.8); to prove the previous one we notice that by $(2.9)$ we have $\left\|f_{\mathcal{E}_{k} \backslash \mathcal{E}_{k+1}}\right\|_{L^{\infty}\left(\ell^{q}\right)} \leq 2^{k+1}$ and the definition of $\dot{\mathbf{g}}_{\infty}^{q^{\prime},(1)}$ implies that $\left\|g_{\mathcal{E}_{k} \backslash \mathcal{E}_{k+1}}\right\|_{L^{1}\left(\ell^{q^{\prime}}\right)} \leq\left\|g_{\mathcal{E}_{k}}\right\|_{L^{1}\left(\ell^{q^{\prime}}\right)} \leq\left|E_{k}\right|$. So, (2.5) is proven. 
Let us now prove (2.6). Let $\varphi$ be a bounded linear functional on $\dot{\mathbf{g}}_{1}^{q}$. By the Hahn-Banach theorem it can be extended to a functional on $L^{1}\left(\ell^{q}\right)$, so it can be represented as

$$
\varphi(f)=\langle f, \widetilde{g}\rangle=\int_{\mathbb{R}} \sum_{k \in \mathbb{Z}} f(x, k) \widetilde{g}(x, k) d x
$$

where $\widetilde{g} \in L^{\infty}\left(\ell^{q^{\prime}}\right),\|\widetilde{g}\|_{L^{\infty}\left(\ell q^{\prime}\right)}=\|\varphi\|$.

Let $g=\mathcal{P}_{\text {seq }}(\widetilde{g})$ be the projection of $\widetilde{g}$ onto the space of sequences, i.e., let the function $g$ be defined by (2.4). The projection $\mathcal{P}_{\text {seq }}$, as can be shown easily, is not bounded in $L^{\infty}\left(\ell^{q^{\prime}}\right)$, but by the Fefferman-Stein theorem (Theorem 2.1) it is bounded in $L^{r}\left(\ell^{q^{\prime}}\right)$.

Therefore, for any (finite union of intervals) $E \subset \mathbb{R}$ and the collection $\mathcal{E}:=$ $\{I \in \mathcal{L}: I \subset E\}$,

$$
\left\|g_{\mathcal{E}}\right\|_{L^{r}\left(\ell^{q^{\prime}}\right)}^{r} \leq C\left\|\widetilde{g}_{\mathcal{E}}\right\|_{L^{r}\left(\ell q^{\prime}\right)}^{r} \leq C|E|\left\|\widetilde{g}_{\mathcal{E}}\right\|_{L^{\infty}\left(\ell q^{\prime}\right)}^{r},
$$

which means exactly that $g \in \dot{\mathbf{g}}_{\infty}^{q^{\prime},(r)},\|g\|_{\dot{\mathbf{g}}_{\infty}^{q^{\prime},(r)}} \leq C\|\varphi\|$.

\subsection{Spaces $\dot{\mathrm{g}}_{\infty}^{q,(r)}$ for $r<1$}

Remark 2.5. In fact, any $r \in(0, \infty)$ can be used in the definition of $\mathbf{g}_{\infty}^{q}$, i.e., all the "norms" $\|\cdot\|_{\dot{\mathbf{g}}_{\infty}^{q,(r)}}, r \in(0, \infty)$ are equivalent (of course, for $r<1$ the quantity $\|\cdot\|_{\dot{\mathbf{g}}_{\infty}^{q,(r)}}$ is not a norm).

This can be proved using the standard John-Nirenberg type estimates, but we present a different argument, based on a simple lemma below.

Lemma 2.6 (Extrapolation of inverse Hölder inequality). Let $\mu$ be a probability measure, and let $L^{p}=L^{p}(\mu)$. For a function $f \in L^{p_{1}}$ and for some $p_{2}>p_{1}$, suppose that there holds the following reverse Hölder inequality:

$$
\|f\|_{p_{2}} \leq C\|f\|_{p_{1}} .
$$

Then, for any $p<p_{1}$,

$$
\|f\|_{p_{1}} \leq C^{\beta}\|f\|_{p}, \quad \beta=\frac{p_{2}}{p} \cdot \frac{p_{1}-p}{p_{2}-p_{1}} .
$$

Proof. Define $r:=\left(p_{2}-p\right) /\left(p_{2}-p_{1}\right)$. Then the dual Hölder exponent is given by $r^{\prime}:=\left(p_{2}-p\right) /\left(p_{1}-p\right)$. The exponent $r$ is chosen so

$$
\frac{1}{r} \cdot p+\frac{1}{r^{\prime}} \cdot p_{2}=p_{1} \text {. }
$$

Assume without loss of generality that $f \geq 0$. Setting $\alpha=p / r$ we can write

$$
\begin{array}{rlrl}
\|f\|_{p_{1}}^{p_{1}}=\left\|f^{p_{1}}\right\|_{1} & \leq\left\|f^{\alpha}\right\|_{r}\left\|f^{p_{1}-\alpha}\right\|_{r^{\prime}} & & \text { by the Hölder inequality } \\
& =\|f\|_{p}^{\alpha}\|f\|_{p_{2}}^{p_{1}-\alpha} & \\
& \leq\|f\|_{p}^{\alpha}\left(C\|f\|_{p_{1}}\right)^{p_{1}-\alpha} & & \text { by }(2.11) .
\end{array}
$$


The last inequality implies that

$$
\|f\|_{p_{1}}^{\alpha} \leq C^{p_{1}-\alpha}\|f\|_{p}^{\alpha}
$$

which gives us the conclusion of the lemma with $\beta=\left(p_{1}-\alpha\right) / \alpha$. To see that it is the same $\beta$ as in the lemma, recall that $\alpha=p / r$, so by (2.12) we have $p_{1}-\alpha=p_{2} / r^{\prime}$. Therefore

$$
\beta=\frac{p_{1}-\alpha}{\alpha}=\frac{p_{2}}{r^{\prime}} \cdot \frac{r}{p}=\frac{p_{2}}{p} \cdot \frac{p_{1}-p}{p_{2}-p_{1}}
$$

To show the equivalence of the norms $\|\cdot\|_{\dot{\mathbf{g}}_{\infty}^{q,(r)}}$ for all $r \in(0, \infty)$, take $r_{2}>$ $r_{1} \geq 1>r>0$. We have proved that the norms $\|\cdot\|_{\dot{\mathbf{g}}_{\infty}^{q,\left(r_{1}\right)}}$ and $\|\cdot\|_{\dot{\mathbf{g}}_{\infty}^{q,\left(r_{2}\right)}}$ are equivalent, i.e., there exists $C=C\left(r_{1}, r_{2}\right)$ such that, for all sequences $s=\left\{s_{I}\right\}_{I \in \mathcal{L}}$,

$$
\|s\|_{\dot{\mathbf{g}}_{\infty}^{q,\left(r_{1}\right)}} \leq\|s\|_{\dot{\mathbf{g}}_{\infty}^{q,\left(r_{2}\right)}} \leq C\|s\|_{\dot{\mathbf{g}}_{\infty}^{q,\left(r_{1}\right)}}
$$

(the first inequality here is Hölder inequality).

Take $\varepsilon>0$ and let $J \in \mathcal{L}$ be such that

$$
\left\|\left(|J|^{-1} \sum_{I \in \mathcal{L}: I \subset J}\left|s_{I}\right|^{2} \mathbf{1}_{I}\right)^{1 / 2}\right\|_{r_{1}} \geq(1+\varepsilon)^{-1}\|s\|_{\dot{\mathbf{g}}_{\infty}^{q,\left(r_{1}\right)}} .
$$

Combining this inequality with (2.13) we get

$$
\begin{aligned}
\left\|\left(|J|^{-1} \sum_{I \in \mathcal{L}: I \subset J}\left|s_{I}\right|^{2} \mathbf{1}_{I}\right)^{1 / 2}\right\|_{r_{2}} & \leq\|s\|_{\dot{\mathbf{g}}_{\infty}^{q,\left(r_{2}\right)}} \leq C\|s\|_{\dot{\mathbf{g}}_{\infty}^{q,\left(r_{1}\right)}} \\
& \leq(1+\varepsilon) C\left\|\left(|J|^{-1} \sum_{I \in \mathcal{L}: I \subset J}\left|s_{I}\right|^{2} \mathbf{1}_{I}\right)^{1 / 2}\right\|_{r_{1}},
\end{aligned}
$$

i.e., we have the inverse Hölder inequality assumption of Lemma 2.6. Therefore, by Lemma 2.6 ,

$$
\begin{aligned}
\left\|\left(|J|^{-1} \sum_{I \in \mathcal{L}: I \subset J}\left|s_{I}\right|^{2} \mathbf{1}_{I}\right)^{1 / 2}\right\|_{r_{1}} & \leq(1+\varepsilon)^{\beta} C^{\beta}\left\|\left(|J|^{-1} \sum_{I \in \mathcal{L}: I \subset J}\left|s_{I}\right|^{2} \mathbf{1}_{I}\right)^{1 / 2}\right\|_{r} \\
& \leq(1+\varepsilon)^{\beta} C^{\beta}\|s\|_{\dot{\mathbf{g}}_{\infty}^{q,(r)}}
\end{aligned}
$$

Combining this estimate with (2.14) we get that

$$
\|s\|_{\dot{\mathbf{g}}_{\infty}^{q,\left(r_{1}\right)}} \leq(1+\varepsilon)^{\beta+1} C^{\beta}\|s\|_{\dot{\mathbf{g}}_{\infty}^{q,(r)}},
$$

which gives the equivalence of $\|s\|_{\dot{\mathbf{g}}_{\infty}^{q,(r)}}$ and $\|s\|_{\dot{\mathbf{g}}_{\infty}^{q,\left(r_{1}\right)}}$ (the opposite inequality $\|s\|_{\dot{\mathbf{g}}_{\infty}^{q,(r)}} \leq\|s\|_{\dot{\mathbf{g}}_{\infty}^{q,\left(r_{1}\right)}}$ follows immediately from the Hölder inequality). 


\subsection{Embedding theorem for $\dot{\mathrm{g}}_{p}^{q}$}

Let $\left\{\alpha_{I}\right\}_{I \in \mathcal{L}}$ be a collection of numbers. We want to know when the operator

$$
f \mapsto\left\{\alpha_{I}\langle f\rangle_{I}\right\}_{I \in \mathcal{L}}
$$

is a bounded operator from $L^{p}$ to $\dot{\mathbf{g}}_{p}^{q}$; recall that for a function $f$ the symbol $\langle f\rangle_{I}$ denotes its average, $\langle f\rangle_{I}=f_{I} f$.

The answer to this question is well known if $p=q$, and is given by the famous Carleson embedding theorem, that says that a necessary and sufficient condition for the boundedness is

$$
\sup _{I \in \mathcal{L}} \frac{1}{|I|} \sum_{J \in \mathcal{L}: J \subset I}\left|\alpha_{J}\right|^{q}<\infty,
$$

which means exactly that $\left\{\alpha_{I}\right\}_{I \in \mathcal{L}} \in \dot{\mathbf{g}}_{\infty}^{q,(q)}=\dot{\mathbf{g}}_{\infty}^{q}$. This result is especially well known for $p=q=2$; the situation for $p=q$ can be obtained, as it will be shown below, by a standard comparison with the maximal function.

Below, we will show that the answer is the same for all $p \in(1, \infty)$. For $p=1$ the above condition is not sufficient for the embedding, but if we replace $L^{1}$ by $\widetilde{H}^{1}$, then the result can be extended to $p=1$.

Theorem 2.7. Let $p \in[1, \infty)$ and $q \in(1, \infty)$, and let $\alpha=\left\{\alpha_{I}\right\}_{I \in \mathcal{L}}$ be a collection of numbers. Then the operator $A_{\alpha}$ defined by

$$
A_{\alpha} f=\left\{\alpha_{I}\langle f\rangle_{I}\right\}_{I \in \mathcal{L}}
$$

is a bounded operator $\widetilde{H}^{p} \rightarrow \mathbf{g}_{p}^{q}$ if and only if $\alpha \in \mathbf{\mathbf { g }}_{\infty}^{q}$.

Proof. To prove the necessity of the condition $\alpha \in \mathbf{g}_{\infty}^{q}$ for $p>1$ we just have to test the embedding operator on the functions $\mathbf{1}_{I}, I \in \mathcal{L}$. Since $\left\langle\mathbf{1}_{I}\right\rangle_{J}=1$ for $J \subset I$, and since for $p \in(1, \infty)$ the $\widetilde{\mathcal{H}}^{p}$-norm is equivalent to the $L^{p}$ norm (see Section 1.3), the boundedness of the operator $A_{\alpha}$ implies

$$
\left\|\left(\sum_{J \in \mathcal{L}: J \subset I} \mid \alpha_{J}{ }^{q} \mathbf{1}_{J}\right)^{1 / q}\right\|_{p}^{p} \leq C\left\|\mathbf{1}_{I}\right\|_{p}^{p}=C|I|,
$$

which means $\alpha \in \dot{\mathbf{g}}_{\infty}^{q,(p)}=\dot{\mathbf{g}}_{\infty}^{q}$ (by Theorem 2.3 the space $\dot{\mathbf{g}}_{\infty}^{q,(p)}$ does not depend on $p$ ).

For $p=1$ we need a bit more complicated test function, since generally $\mathbf{1}_{I}$ is not in $\widetilde{\mathcal{H}}^{1}$. To prove the necessity in this case, take $I \in \mathcal{L}$. We need to consider two possibilities:

(i) $|K|<2|I|$ for all $K \in \mathcal{L}, K \supset I$.

(ii) There exists $K \in \mathcal{L}, K \supset I$ such that $|K| \geq 2|I|$.

In the first case

$$
\left|\operatorname{supp}\left(M_{\mathcal{L}} \mathbf{1}_{I}\right)\right| \leq 2|I|, \quad \text { so } \quad\left\|M_{\mathcal{L}} \mathbf{1}_{I}\right\|_{1} \leq 2|I| .
$$


The maximal function characterization of $\widetilde{H}^{1}$ (Theorem 1.2) implies that $\mathbf{1}_{I} \in \widetilde{H}^{1}$, $\left\|\mathbf{1}_{I}\right\|_{\widetilde{H}^{1}} \leq C|I|$, so testing as above the operator $A_{\alpha}$ on $f=\mathbf{1}_{I}$ we get

$$
\left\|\left(\sum_{J \in \mathcal{L}: J \subset I}\left|\alpha_{J}\right|{ }^{q} \mathbf{1}_{J}\right)^{1 / q}\right\|_{1} \leq C\|f\|_{\widetilde{H}^{1}} \leq C|I| .
$$

In the second case, let $K \in \mathcal{L}$ be the first ancestor of $I$ such that $2|I| \leq|K|$. Let $f:=\mathbf{1}_{I}+\alpha \mathbf{1}_{K \backslash I}$, where $\alpha \in \mathbb{R}$ is chosen such, that $\int f d x=0$. It is easy to check, using again the maximal function characterization of $\widetilde{H}^{1}$ (Theorem 1.2), that $f \in H^{1}$ and $\|f\|_{\widetilde{H}^{1}} \leq C|I|$. Since for $J \in \mathcal{L}, J \subset I$ we still have $\langle f\rangle_{J}=1$, testing $A_{\alpha}$ on $f$ we get the estimate (2.15).

Let us now prove sufficiency. Let

$$
E_{k}=\left\{x \in \mathbb{X}:\left|M_{\mathcal{L}} f(x)\right|>2^{k}\right\}, \quad \text { and } \quad \mathcal{E}_{k}=\left\{I \in \mathcal{L}: I \subset E_{k}\right\} .
$$

We can write

$$
A_{\alpha} f=\sum_{k \in \mathbb{Z}}\left(A_{\alpha} f\right)_{\mathcal{E}_{k} \backslash \mathcal{E}_{k+1}}
$$

where $\left(A_{\alpha} f\right)_{\mathcal{E}_{k} \backslash \mathcal{E}_{k+1}}$ denotes the coordinate projection of $A_{\alpha} f$, see (2.1), with $\mathcal{E}=\mathcal{E}_{k} \backslash \mathcal{E}_{k+1}$.

Assume that $\|\alpha\|_{\dot{\mathbf{g}}_{\infty}^{q}} \leq 1$, and let $\alpha_{\mathcal{E}_{k} \backslash \mathcal{E}_{k+1}}$ be the corresponding coordinate projection (2.1) of $\alpha$. Since $\left|\mathbb{E}_{I} f\right| \leq\left|M_{\mathcal{L}} f\right| \leq 2^{k+1}$ on $I \in \mathcal{E}_{k} \backslash \mathcal{E}_{k+1}$, we conclude that

$$
\left\|\left(A_{\alpha} f\right)_{\mathcal{E}_{k} \backslash \mathcal{E}_{k+1}}\right\|_{L^{p}\left(\ell^{q}\right)}^{p} \leq 2^{(k+1) p}\left\|\alpha_{\mathcal{E}_{k} \backslash \mathcal{E}_{k+1}}\right\|_{L^{p}\left(\ell^{q}\right)}^{p} \leq 2^{(k+1) p}\left|E_{k}\right| .
$$

Therefore,

$$
\sum_{k \in \mathbb{Z}}\left\|\left(A_{\alpha} f\right)_{\mathcal{E}_{k} \backslash \mathcal{E}_{k+1}}\right\|_{L^{p}\left(\ell^{q}\right)}^{p} \leq \sum 2^{(k+1) p}\left|E_{k}\right| \leq C\left\|M_{\mathcal{L}} f\right\|_{p}^{p} \leq C_{1}\|f\|_{\widetilde{H}^{p}}^{p}
$$

here the second inequality follows from Lemma 2.4, and the last one is the boundedness of the maximal function in $L^{p}$ for $p>1$.

The estimate $(2.16)$ is exactly what we need if $p=q$.

The case $p<q$ is also easy. We can write (in the functional representation)

$$
\begin{aligned}
\left\|A_{\alpha} f\right\|_{L^{p}\left(\ell^{q}\right)}^{p} & =\int_{\mathbb{X}}\left(\sum_{k \in \mathbb{Z}}\left\|\left(A_{\alpha} f\right)_{\mathcal{E}_{k} \backslash \mathcal{E}_{k+1}}(x, \cdot)\right\|_{\ell^{q}}^{q}\right)^{\frac{1}{q} p} d x \\
& \leq \int_{\mathbb{X}}\left(\sum_{k \in \mathbb{Z}}\left\|\left(A_{\alpha} f\right)_{\mathcal{E}_{k} \backslash \mathcal{E}_{k+1}}(x, \cdot)\right\|_{\ell^{q}}^{p}\right)^{\frac{1}{p} p} d x \quad\|s\|_{\ell^{q}} \leq\|s\|_{\ell^{p}}, p<q \\
& =\sum_{k \in \mathbb{Z}}\left\|\left(A_{\alpha} f\right)_{\mathcal{E}_{k} \backslash \mathcal{E}_{k+1}}\right\|_{L^{p}\left(\ell^{q}\right)}^{p} \leq C\|f\|_{\widetilde{H}^{p}}^{2} \quad \text { by }(2.16) .
\end{aligned}
$$

The case $p>q$ is a bit more complicated. To treat this case let us first make some simplifications. Of course, without loss of generality we can assume that $f \geq 0$ and that all $\alpha_{I} \geq 0$. 
Lemma 2.8. Let $f \in{ }_{\sim} L^{p}, 1<p<\infty$, and $f \geq 0$. There exists a function $\tilde{f}$ satisfying $\tilde{f} \geq f$ and $\|\widetilde{f}\|_{p} \leq C_{p}\|f\|_{p}$ such that

$$
M \tilde{f} \leq C_{p}^{\prime} \tilde{f}
$$

where $M$ is the Hardy-Littlewood maximal function.

The condition $M f \leq C f$ for $f \geq 0$ is the so-called Muckenhoupt $\left(A_{1}\right)$ condition. It implies, in particular, that for any interval $I$,

$$
f_{I} f \leq \frac{1}{C} \min _{x \in I} f(x)
$$

Proof of Lemma 2.8. Define $\tilde{f}$ by

$$
\widetilde{f}:=\sum_{k \geq 0} \gamma^{k} M^{k} f,
$$

where $M^{k}$ is $k$ th iteration of $M$, and $\gamma>0$ is sufficiently small, so that

$$
\gamma\|M h\|_{p} \leq \frac{1}{2}\|h\|_{p} \quad \forall h \in L^{p} .
$$

Replacing $f$ by the $\tilde{f}$ from Lemma 2.8 we can assume without loss of generality that $M f \leq C f$.

It is an easy exercise with the Hölder inequality and the Resonance Lemma (the fact that equality is attained in Hölder's inequality) to see that if $p>q$ and $1 / p+1 / r=1 / q$,

$$
\|F\|_{p}=\sup \left\{\|F g\|_{q}: g \in L^{r},\|g\|_{r} \leq 1\right\} .
$$

Take $g \in L^{r}$ such that $g \geq 0$ and $\|g\|_{r} \leq 1$. Since clearly $r>q$, applying Lemma 2.8 to $g^{q}$ with the exponent $s=r / q$ for $p$, we get a function $\widetilde{g} \geq g$ such that $M\left(\widetilde{g}^{q}\right) \leq C \widetilde{g}^{q}$ and

$$
\|\widetilde{g}\|_{r}^{r}=\left\|\widetilde{g}^{q}\right\|_{s}^{s} \leq C\left\|g^{q}\right\|_{s}^{s}=C\|g\|_{r}^{r} .
$$

So, replacing $g$ by $\widetilde{g}$ we can assume without loss of generality that $M g \leq C g$ and $\|g\|_{r} \leq C$.

To complete the proof, let us first notice that

$$
\left\|A_{\alpha}(f g)\right\|_{L^{q}\left(\ell^{q}\right)} \leq C\|f g\|_{q} \leq C\|f\|_{p}\|g\|_{r} \leq C^{\prime}\|f\|_{p} .
$$

This inequality follows from the case $p=q$ we discussed above. We used here the fact that $\|\alpha\|_{\dot{\mathbf{g}}_{\infty}^{q,(q)}} \leq\|\alpha\|_{\dot{\mathbf{g}}_{\infty}^{q,(p)}}$, which follows immediately from Hölder's inequality; note that we do not need here the full equivalence of the $\dot{\mathbf{g}}_{\infty}^{q,(p)}$-norms for all $p$.

So, in light of $(2.17)$, we only need to show that

$$
\left\|\left(A_{\alpha} f\right) g\right\|_{L^{q}\left(\ell^{q}\right)} \leq C\left\|A_{\alpha}(f g)\right\|_{L^{q}\left(\ell^{q}\right)},
$$


which follows immediately if the estimate

$$
f_{I}\left|\langle f\rangle_{I} g\right|^{q} \leq C\langle f g\rangle_{I}^{q} .
$$

holds uniformly for all $I \in \mathcal{L}$. We know that

$$
\min _{x \in I} f(x) \leq\langle f\rangle_{I} \leq C \min _{x \in I} f(x)
$$

and that

$$
\left\langle g^{q}\right\rangle_{I} \leq C \min _{x \in I} g^{q}(x)=C\left(\min _{x \in I} g(x)\right)^{q} \leq C\langle g\rangle_{I}^{q}
$$

and therefore

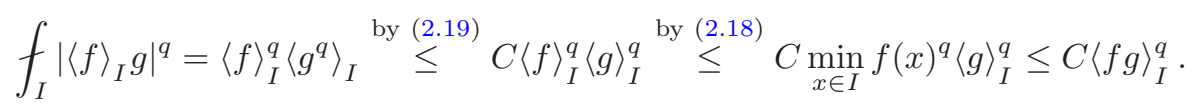

\section{3. $\mathcal{H}_{q}^{p}$ and $\mathcal{B M O}_{q}$ spaces}

Most of the results of this section are well known, and are presented here only for the convenience of the reader. However, I believe some of the proofs are new.

\section{1. $\mathcal{H}_{q}^{p}$ spaces}

As we discussed in Section 1.3, the $L^{p}$ norm of a function $f, p \in(1, \infty)\left(H^{1}\right.$ norm if $p=1$ ), is equivalent to the $L^{p}$ norm of the square function $S f$.

Acting by analogy, one can use the " $q$-function" instead, and consider the space $\widetilde{\mathcal{H}}_{q}^{p}, p, q \in[1, \infty]$, of formal martingale difference decompositions, such that

$$
\|f\|_{\widetilde{\mathcal{H}}_{q}^{p}}:=\left\|\left(\sum_{I \in \mathcal{L}}\left|\Delta_{I} f\right|^{q}+\sum_{I \in \mathfrak{A}_{-\infty}^{0, \text { fin }}}\left|\mathbb{E}_{I} f\right|^{q}\right)^{1 / q}\right\|_{L^{p}}<\infty
$$

We assume here that the "martingale differences" $\Delta_{I} f$ are simply some functions $h_{I}$, constant on children of $I$ and such that $\int_{\mathbb{X}} h_{I} d x=0$. The functions $E_{I} f$ are just some multiples of $\mathbf{1}_{I}$. While we do not assume that all the $h_{I}$ are martingale differences for some function $f$, we will still use the notation $\Delta_{f}$, meaning by $f$ the whole collection of such "martingale differences". ${ }^{1}$

We can also consider the space $\mathcal{H}_{q}^{p}$, consisting of formal martingale difference decompositions for which $\mathbb{E}_{I}=0$ for all $I \in \mathfrak{A}_{-\infty}^{0, \text { fin }}$.

The spaces $\widetilde{\mathcal{H}}_{q}^{p}$ and $\mathcal{H}_{q}^{p}$ are clearly Banach spaces, as closed subspaces of $L^{p}\left(\ell^{q}\right)$.

As we discussed above in Section 1.3, if $f \in \widetilde{\mathcal{H}}_{2}^{p}$, then the series converges to a function in $L^{p}$ (to a function in $\widetilde{H}^{1}$ if $p=1$ ), and for $p \in(1, \infty)$ the norm $\|f\|_{\widetilde{\mathcal{H}}_{2}^{p}}$ is equivalent to the standard $L^{p}$ norm.

\footnotetext{
${ }^{1}$ Such notation is partially justified by the fact that, in the essential case where our collection has only finitely many nonzero terms, all $\Delta_{f}$ are martingale differences of the function $\sum_{I \in \mathcal{L}} \Delta_{I} f$.
} 
Since $\|x\|_{\ell^{2}} \leq\|x\|_{\ell^{q}}$ for $q \in[1,2]$, any formal martingale decomposition $f \in \mathcal{H}_{q}^{p}$, $q \in[1,2]$, converges to a function in $L^{p}$. Thus, in this case we can identify the spaces $\widetilde{\mathcal{H}}_{q}^{p}$ and $\mathcal{H}_{q}^{p}$ with function spaces, which we denote by $\widetilde{H}_{q}^{p}$ and $H_{q}^{p}$, respectively.

For $q>2$ convergence is not clear, so in this case we only consider the spaces $\widetilde{\mathcal{H}}_{q}^{p}$ and $\mathcal{H}_{q}^{p}$ of formal martingale differences.

Remark 3.1. Informally, we can say that $f \in \mathcal{H}_{q}^{p}$ if $\left\{\Delta_{I} f\right\}_{I \in \mathcal{L}} \in \dot{\mathbf{g}}_{p}^{q}$. We are saying "informally" here, because the $\Delta_{f}$ are not numbers but functions, so we have to interpret the sequence $\left\{\Delta_{I} f\right\}_{I \in \mathcal{L}}$ as a sequence of numbers.

In this paper we will interpret this by saying that each $\Delta_{I} f$ determines the number $x_{J}, J \in \operatorname{child}(I)$, where $x_{J}$ is simply the value of $\Delta_{I} f$ on $J$.

The space $\mathcal{H}_{2}^{1}$ is the classical martingale $H^{1}$ space, and we will often skip the index $q$ referring to the case $q=2$. The spaces $\mathcal{H}_{q}^{p}$ were studied by many authors; for example the spaces $H_{p}^{S_{q}}$ were considered in [15] (the superscript stands for "square function").

Remark. There are alternative ways of obtaining the numbers $x_{I}$ from the martingale differences $\Delta_{I}$. For example, one puts $x_{I}:=\left(\mathbb{E}_{I}\left|\Delta_{I} f\right|^{q}\right)^{1 / q}$, and the condition $\left\{x_{I}\right\}_{I \in \mathcal{L}} \in \dot{\mathbf{g}}_{p}^{q}$, defines a martingale Hardy space that is denoted by $H_{p}^{s_{q}}$ in [15] (note that the superscript here is $s_{q}$, no the $S_{q}$ of the previous paragraph).

For homogeneous lattices it is not hard to show that this definition is equivalent to the first one. It is also well known and can be seen from what follows, that in the general, non-homogeneous case, these spaces can be different.

\subsection{BMO spaces and $H^{1}-B M O$ duality}

We want to define BMO spaces, so we have the $H^{1}$-BMO duality, as usual.

Definition. We say that a formal martingale difference decomposition

$$
f=\sum_{I \in \mathcal{L}} \Delta_{I} f
$$

belongs to the space $\mathcal{B M O}_{q}, q \in(1, \infty)$ if the sequence $\left\{x_{I}\right\}_{I \in \mathcal{L}}$, obtained from $\left\{\Delta_{I} f\right\}_{I \in \mathcal{L}}$ as in Remark 3.1, belongs to $\dot{\mathbf{g}}_{\infty}^{q}$.

The extended BMO spaces $\mathcal{B M O}_{q}^{\sim}$ are obtained by adding to the formal sum $f \in \mathcal{B M O}_{q}$ additional terms

$$
\sum_{I \in \mathfrak{A}_{-\infty}^{0, \text { fin }}} \mathbb{E}_{I} f, \quad\left\|\mathbb{E}_{I} f\right\|_{\infty} \leq C<\infty \quad \forall I \in \mathfrak{A}_{-\infty}^{0, \text { fin }} .
$$

We can rewrite the definition of $\mathcal{B M O}_{q}$ by picking $r \in(0, \infty)$ and saying that $f \in \mathcal{B M O}_{q}$ if, for any $I \in \mathcal{L}$,

$$
f_{I}\left(\sum_{J \in \mathcal{L}: J \subset I}\left|\Delta_{J} f\right|^{q}\right)^{\frac{1}{q} r} d x \leq C<\infty
$$


(uniformly in $I$ ), and, in addition

$$
\sup _{I \in \mathcal{L}}\left\|\Delta_{I} f\right\|_{\infty}<\infty
$$

These two conditions mean exactly that the sequence $x=\left\{x_{I}\right\}_{I \in \mathcal{L}}$, obtained from $\left\{\Delta_{I} f\right\}_{I \in \mathcal{L}}$ as in Remark 3.1, belongs to $\dot{\mathrm{g}}_{\infty}^{q,(r)}$. Since, as we discussed above (see Theorem 2.3 and Remark 2.5) the spaces $\dot{\mathbf{g}}_{\infty}^{q,(r)}, r \in(0, \infty)$ coincide, this means $x \in \dot{\mathbf{g}}_{\infty}^{q,(q)}=: \dot{\mathbf{g}}_{\infty}^{q}$.

Remark. One would expect that the condition (3.2) alone defines the space $\mathcal{B M O}_{q}$, but it has been known for a long time that the additional condition is needed. One can look, for example, at Garsia's 1973 book [7], where the $\mathcal{B M O}_{2}$ space was defined. One can easily see that the definition from [7] is equivalent to the one presented here.

If $|\mathbb{X}|<\infty, \mathcal{B M O}_{q} \subset \mathcal{H}_{q}^{r}$, so (see Section 3.1 above) for $q \in(1,2]$ the formal martingale difference decomposition $f \in \mathcal{B M O}_{q}$ converges to a function in $L^{r}$. So in the case $|\mathbb{X}|<\infty$ one can identify for $q \in[1,2]$ the spaces $\mathcal{B M O}_{q}$ and $\mathcal{B M O}_{q}^{\sim}$ with function spaces, which we will call $\mathrm{BMO}_{q}$ and $\mathrm{BMO}_{q}^{\sim}$, respectively.

The following theorem is known, but the proof presented here is probably new.

Theorem 3.2. The dual of the space $\mathcal{H}_{q}^{1}$ (respectively, $\widetilde{\mathcal{H}}_{q}^{1}$ ), $1<q<\infty$, is the space $\mathcal{B M O}_{q^{\prime}}$ (respectively, $\mathcal{B M O}_{q^{\prime}}^{\sim}$ ).

Proof. We will prove the duality between $\mathcal{H}_{q}^{1}$ and $\mathcal{B M O}_{q^{\prime}}$. The duality between $\widetilde{\mathcal{H}}_{q}^{1}$ and $\mathcal{B} \mathcal{M O} \mathcal{O}_{q^{\prime}}^{\sim}$ follows trivially.

The sufficiency of the condition $g \in \mathcal{B M O}_{q^{\prime}}$ for the boundedness of the linear functional $f \mapsto\langle f, g\rangle$ on $\mathcal{H}_{q}^{1}$ follows immediately from Theorem 2.3.

To prove the necessity of this condition, let us note that by definition $\mathcal{H}_{1}^{q}$ can be identified with a subspace of $\dot{\mathbf{g}}_{1}^{q}$ (by identifying the family $\left\{\Delta_{I} g\right\}_{I \in \mathcal{L}}$ with an element in $\dot{\mathbf{g}}_{1}^{q}$ as described in Remark 3.1).

Thus a linear functional $\varphi$ on $\mathcal{H}_{q}^{1}$ can be extended by the Hahn-Banach theorem to a functional on $\dot{\mathbf{g}}_{1}^{q}$, so by Theorem 2.3 there exists $\widetilde{g}=\left\{\widetilde{g}_{I}\right\}_{I \in \mathcal{L}} \in \dot{\mathbf{g}}_{\infty}^{q^{\prime}},\|\widetilde{g}\|_{\dot{\mathbf{g}}_{\infty}^{q^{\prime}}} \leq$ $C\|\varphi\|$, such that the functional $\varphi$ is given by

$$
\varphi(f)=\int_{\mathbb{X}} \sum_{I \in \mathcal{L}} \sum_{J \in \operatorname{child}(I)} \Delta_{I} f(x) \widetilde{g}_{J} d x=\sum_{I \in \mathcal{L}} \int_{\mathbb{X}} \sum_{J \in \operatorname{child}(I)} \Delta_{I} f(x) \widetilde{g}_{J} d x .
$$

We would like to interpret the function $\sum_{J \in \operatorname{child}(I)} \widetilde{g}_{J} \mathbf{1}_{J}$ as a martingale difference, but this function does not have average zero. But since $\int_{\mathbb{X}} \Delta_{I} f d x=0$, the integrals on the right side of (3.4) do not change if we subtract from $\widetilde{g}_{J}, J \in \operatorname{child}(I)$, a constant $c=c_{I}$.

Therefore, if for $J \in \operatorname{child}(I)$ we define $g_{J}:=\widetilde{g}_{J}-|I|^{-1} \sum_{I^{\prime} \in \operatorname{child}(I)} \widetilde{g}_{I^{\prime}}\left|I^{\prime}\right|$, we get that

$$
\varphi(f)=\sum_{I \in \mathcal{L}} \int_{\mathbb{X}} \sum_{J \in \operatorname{child}(I)} \Delta_{I} f(x) g_{J} d x
$$


However now the functions $\sum_{J \in \operatorname{child}(I)} g_{J} \mathbf{1}_{J}$ have zero average, so we can treat them as martingale differences.

Let us check that $\left\{g_{I}\right\}_{I \in \mathcal{L}} \in \dot{\mathbf{g}}_{\infty}^{q^{\prime}}$. Using the fact that the averaging operator $f \mapsto\langle f\rangle_{I} \mathbf{1}_{I}$ is a contraction in all $L^{p}, p \in[1, \infty]$ (it follows immediately from the Hölder inequality), we can see that

$$
\left\|\sum_{J \in \operatorname{child}(I)} g_{J} \mathbf{1}_{J}\right\|_{q^{\prime}} \leq 2\left\|\sum_{J \in \operatorname{child}(I)} \widetilde{g}_{J} \mathbf{1}_{J}\right\|_{q^{\prime}}
$$

Using this inequality we get that for $I_{0} \in \mathcal{L}$

$$
\begin{aligned}
& \sum_{J \in \mathcal{L}: J \subset I_{0}}\left|g_{J}\right|^{q^{\prime}}|J|=\left|g_{I_{0}}\right| q^{q^{\prime}} \cdot\left|I_{0}\right|+\sum_{I \in \mathcal{L}: I \subset I_{0}}\left\|\sum_{J \in \operatorname{child}(I)} g_{J} \mathbf{1}_{J}\right\|_{q^{\prime}}^{q^{\prime}} \\
& \leq\left|g_{I_{0}}\right|^{q^{\prime}} \cdot\left|I_{0}\right|+\left.2^{q^{\prime}} \sum_{I \in \mathcal{L}: I \subset I_{0}}\left\|\sum_{J \in \operatorname{child}(I)} \widetilde{g}_{J} \mathbf{1}_{J}\right\|\right|_{q^{\prime}} ^{q^{\prime}} \leq\left|g_{I_{0}}\right| q^{q^{\prime}} \cdot\left|I_{0}\right|+2^{q^{\prime}}\left|I_{0}\right| \cdot\|\widetilde{g}\|_{\dot{\mathbf{g}}_{\infty}^{q^{\prime},\left(q^{\prime}\right)}}^{q^{\prime}}
\end{aligned}
$$

Noticing that $\left|\widetilde{g}_{I_{0}}\right| \leq\|\widetilde{g}\|_{\dot{\mathbf{g}}_{\infty}^{q^{\prime},\left(q^{\prime}\right)}}$, and therefore $\left|g_{I_{0}}\right| \leq 2\|\widetilde{g}\|_{\dot{\mathbf{g}}_{\infty}^{q^{\prime},\left(q^{\prime}\right)}}$, and taking into account that $\dot{\mathbf{g}}_{\infty}^{q^{\prime},(r)}$ norms are equivalent for all $r \in[1, \infty)$, we conclude that $\left\|\left\{g_{I}\right\}_{I \in \mathcal{L}}\right\|_{\dot{\mathbf{g}}_{\infty}^{q^{\prime}}} \leq C\|\widetilde{g}\|_{\mathbf{g}_{\infty}^{q^{\prime}}}$. Thus $g=\left\{g_{I}\right\}_{I \in \mathcal{L}} \in \mathcal{B} \mathcal{M} \mathcal{O}_{q^{\prime}}$.

\section{3. $\mathcal{B M O}_{q}$ as function spaces}

Proposition 3.3. For $q \in[1,2]$ the space $\mathcal{B M O}_{q}$ can be identified with a function space, i.e., for each formal martingale decomposition $f=\sum_{I \in \mathcal{L}} \Delta_{I} f \in \mathcal{B M O}_{q}$ there exists a locally integrable function $\tilde{f}$ such that for all $I \in \mathcal{L}$

$$
\Delta_{I} f=\Delta_{I} \tilde{f}
$$

A similar statement holds for the spaces $\mathcal{B M O}_{q}^{\sim}$ as well.

Remark. As can be seen from a simple example below, the martingale difference decomposition $f=\sum_{I \in \mathcal{L}} \Delta_{I} f \in \mathrm{BMO}_{q}, q \in(1,2]$, does not necessarily converge if $|\mathbb{X}|=\infty$.

Let $\mathcal{L}$ be the standard dyadic lattice $\mathcal{D}$ in $\mathbb{R}$, and let $I_{k}=\left[0,2^{k}\right), k \in \mathbb{N}$. Consider the formal martingale sum $f=\sum_{k=1}^{\infty} \Delta_{I_{k}} f$, where

$$
\Delta_{I_{k}} f=\mathbf{1}_{\left[0,2^{k-1}\right)}-\mathbf{1}_{\left[2^{k-1}, 2^{k}\right)} .
$$

It is easy to see that $f \in \mathcal{B M O}_{q}$ for all $q \in(1, \infty)$, but the series clearly diverges.

Proof of Proposition 3.3. It is sufficient to analyze the convergence on each interval $J \in \mathfrak{A}_{-\infty}^{0}$ separately.

If $|J|<\infty$, then the series $f_{J}:=\sum_{I \in \mathcal{L}: I \subset J} \Delta_{I} f$ belongs to $\mathcal{H}_{q}^{2}$. Therefore, as was discussed before in Section 3.1, the series converges to an $L^{2}$ function. 
Let now consider $J \in \mathfrak{A}_{-\infty}^{0}$ such that $|J|=\infty$. It is not hard to see that any such interval can be represented as the union

$$
J=\bigcup_{k \geq 1} I_{k}, \quad I_{k} \in \operatorname{child}\left(I_{k+1}\right) \quad \forall k \geq 1
$$

(note that here $k$ is not the number of generation). Let

$$
\mathcal{L}(J):=\left\{I \in \mathcal{L}: I \subset J, I \neq I_{k} \forall k \in \mathbb{N}\right\}
$$

so the collection $\{I \in \mathcal{L}: I \subset J\}$ is split into a disjoint union of $\mathcal{L}$ and the set $\left\{I_{k},: k \in \mathbb{N}\right\}$.

For $k=2,3, \ldots$ let $\alpha_{k}$ be the value of $\Delta_{I_{k}}$ on $I_{k-1}$, and let $\alpha_{1}=0$. Define the function $\tilde{f}$ on $J$ by

$$
\tilde{f}:=\sum_{I \in \mathcal{L}(J)} \Delta_{I} f+\sum_{s \in \mathbb{N}}\left(\Delta_{I_{s}} f-\alpha_{s} \mathbf{1}_{J}\right)
$$

Let us show that the sum restricted to any of the above intervals $I_{k}$ converges in $L^{2}\left(I_{k}\right)$. This will immediately imply that $\Delta_{I} \tilde{f}=\Delta_{I} f$ for all $I \in \mathcal{L}, I \subset J$.

The second sum converges trivially, because $\Delta_{I_{s}} f-\alpha_{s} \mathbf{1}_{J}=0$ on $I_{k}$ if $s>k$.

Let us show the convergence of the first sum. Note that we only need to count the terms $\Delta_{I} f$ with $I \in \mathcal{L}(J), I \subset I_{k}$, because the terms with $I \in \mathcal{L}(J), I \not \subset I_{k}$ are zero on $I_{k}$.

The condition $f \in \mathcal{B M O}_{q}$ implies that

$$
\sum_{I \in \mathcal{L}(J): I \subset I_{k}} \Delta_{I} f \in \mathcal{H}_{q}^{2} \subset \mathcal{H}_{2}^{2},
$$

so the sum converges in $L^{2}\left(I_{k}\right)$.

Remark 3.4. For $r \in(1, \infty)$, the condition that the sequence obtained from $\left\{\Delta_{I}\right\}_{I \in \mathcal{L}}$ belongs to $\dot{\mathbf{g}}_{\infty}^{2,(r)}$ can be rewritten as

$$
\sup _{I \in \mathcal{L}}|I|^{-1} \int_{I}\left|f-\langle f\rangle_{\widetilde{I}}\right|^{r}<\infty ;
$$

here $\widetilde{I}$ denotes the parent of $I$.

\section{4. $L^{p}$ bounds of paraproducts}

\subsection{Martingale differences do not form a strong unconditional basis in $H^{p}$ in the non-homogeneous case}

In [9] the notion of a strong unconditional basis was introduced. A system of nontrivial subspaces $\mathcal{E}_{j}$ (of a Banach space $X$ ), $j \in \mathcal{J}$ (where $\mathcal{J}$ is a some countable set) was called a strong unconditional basis if

(i) the linear span $\mathcal{L}\left\{\mathcal{E}_{j}: j \in \mathcal{J}\right\}$ is dense in $X$; 
(ii) there exists an ideal Banach space $Y$ of sequences $\left\{c_{j}\right\}_{j \in \mathcal{J}}$ and a constant $A>0$ such that for any sequence $\left\{x_{j}\right\}_{j \in \mathcal{J}}, x_{j} \in \mathcal{E}_{j}$ with finitely many nonzero elements

$$
\frac{1}{A}\left\|\sum_{j \in \mathcal{J}} x_{j}\right\|_{X} \leq\left\|\left\{\left\|x_{j}\right\|\right\}_{j \in \mathcal{J}}\right\|_{Y} \leq A\left\|\sum_{j \in \mathcal{J}} x_{j}\right\|_{X} .
$$

Recall that a Banach space $Y$ of sequences $\left\{c_{j}\right\}_{j \in \mathcal{J}}$ of complex numbers is called ideal if for any sequence of factors $\alpha_{j},\left|\alpha_{j}\right| \leq 1$ the sequence $\left\{\alpha_{j} c_{j}\right\}_{j \in \mathcal{J}} \in Y$ and $\left\|\left\{\alpha_{j} c_{j}\right\}_{j \in \mathcal{J}}\right\|_{Y} \leq\left\|\left\{c_{j}\right\}_{j \in \mathcal{J}}\right\|_{Y}$.

Note that a strong unconditional basis is an unconditional basis, meaning that any vector $x \in X$ admits a unique representation

$$
x=\sum_{j \in \mathcal{J}} x_{j}, \quad x_{j} \in \mathcal{E}_{j},
$$

and the series converges unconditionally, i.e., independently of the ordering of $\mathcal{J}$.

One can easily see that the martingale difference spaces $D_{I}=\Delta_{I}$ form an unconditional basis in $H^{p}, p \in[1, \infty)$. It is also well known that for a homogeneous lattice $\mathcal{L}$ the subspaces $D_{I}$ form a strong unconditional basis.

Unfortunately, as we demonstrate below, that is not the case in the general situation.

If the system of the martingale difference spaces $D_{I}$ were a strong unconditional basis, one might imagine that the natural "coefficient space" for $H^{p}$ should be the Triebel-Lizorkin type space $\mathbf{g}_{p}^{2}$.

In other words, one could guess that one could get an equivalent norm in $H^{p}$ by replacing the functions $\Delta_{I} f$ in the square function by multiples of $\mathbf{1}_{I}$. The norms have to be equivalent on singletons $f=\Delta_{I} f$, so if one wants to replace the functions $\Delta_{I} f$ by $c_{I} \mathbf{1}_{I}, c_{I}=c_{I}(f)$, the functions $\Delta_{I} f$ and $c_{I} \mathbf{1}_{I}$ should have equivalent $L^{p}$ norms (uniformly in $I$ ).

If everything works when the norms of $\Delta_{I} f$ and $c_{I} \mathbf{1}_{I}$ are equivalent, it works when they are equal. So everything reduces to the question of whether the quantity

$$
\left\|\left(\sum_{I \in \mathcal{L}}\left(\mathbb{E}_{I}\left|\Delta_{I} f\right|^{p}\right)^{2 / p}\right)^{1 / 2}\right\|_{p}
$$

gives an equivalent norm on $H^{p}$.

The answer is well known to be "yes" in the case where the lattice is homogeneous. In fact, in this case for $q \in[1, \infty)$ the averages $\left(\mathbb{E}_{I}\left|\Delta_{I}\right|^{q}\right)^{1 / q}$ are equivalent, so one can replace $\Delta_{I} f$ by any of these averages (the case $q=2$ is usually considered in the literature).

In the general case, as the theorem below asserts, only "half" of necessary inequalities are true, so the answer is unfortunately "no".

Note that Theorem 4.1 does not imply that the system of martingale difference spaces $D_{I}$ is not a strong unconditional basis: it only implies that a particular norm on the coefficient space does not give an equivalent norm. However, modifying the proof of Theorem 4.1 one can show that indeed the martingale difference spaces $D_{I}$ do not form a strong unconditional basis in $H^{p}$. 
Theorem 4.1. Let $f \in H^{p}$.

(i) For $p \in[1,2]$ the inequality

$$
\left\|\left(\sum_{I \in \mathcal{L}}\left(\mathbb{E}_{I}\left|\Delta_{I} f\right|^{p}\right)^{2 / p}\right)^{1 / 2}\right\|_{p} \leq C\left\|\left(\sum_{I \in \mathcal{L}}\left|\Delta_{I} f\right|^{2}\right)^{1 / 2}\right\|_{p}
$$

holds; here $C=C_{p}$ and does not depend on $f$ and $\mathcal{L}$.

(ii) For $p \in[2, \infty)$ the opposite inequality

$$
\left\|\left(\sum_{I \in \mathcal{L}}\left|\Delta_{I} f\right|^{2}\right)^{1 / 2}\right\|_{p} \leq C\left\|\left(\sum_{I \in \mathcal{L}}\left(\mathbb{E}_{I}\left|\Delta_{I} f\right|^{p}\right)^{2 / p}\right)^{1 / 2}\right\|_{p}
$$

holds with $C=C_{p}$.

(iii) For $p \in(2, \infty)$ the inequality (4.2) fails, i.e., for each $p>2$ one can find a lattice $\mathcal{L}$ and $f \in H^{p}$ such that the left side of (4.2) is infinite (while the right side is finite because $f \in H^{p}$ ).

(iv) For $p \in[1,2)$ the inequality (4.3) fails (in the same sense as in statement (iii)).

4.1.1. Proof of two of the estimates in Theorem 4.1. To prove statement (i), let us consider the sequence $\left\{\left|\Delta_{I} f\right|^{p}\right\}_{I \in \mathcal{L}} \in \dot{\mathbf{g}}_{1}^{q}, q=2 / p$, where as in Remark 3.1, $\left|\Delta_{I} f\right|^{p}$ defines numbers $x_{J}, J \in \operatorname{child}(I), x_{J}$ being the value of $\left|\Delta_{J} f\right|^{p}$ on $J$. Then the estimate (4.2) follows immediately from the boundedness of the averaging operator $\mathrm{Av}$ in $\dot{\mathbf{g}}_{1}^{q}, q=2 / p$,

$$
(\operatorname{Av} x)_{I}=|I|^{-1} \sum_{J \in \operatorname{child}(I)} x_{J}|J| .
$$

To prove that Av is bounded, let us notice that its adjoint $A v$ * is the "forward shift"

$$
\left(\operatorname{Av}^{*} x\right)_{I}=x_{\widetilde{I}}, \quad \widetilde{I} \text { is a parent of } I .
$$

We want to show that this operator is bounded in $\mathbf{g}_{\infty}^{q^{\prime}}=\left(\mathbf{g}_{1}^{q}\right)^{*}$. If $x=$ $\left\{x_{I}\right\}_{I \in \mathcal{L}} \in \dot{\mathbf{g}}_{\infty}^{q}$, then for $J \in \mathcal{L}$

$$
\sum_{I \in \mathcal{L}: I \subset J}\left|\left(\mathrm{Av}^{*} x\right)_{I}\right|^{q^{\prime}} \mathbf{1}_{I}=\left|x_{\widetilde{J}}\right|^{q^{\prime}} \mathbf{1}_{J}+\sum_{I \in \mathcal{L}: I \subset J}\left|x_{I}\right|^{q^{\prime}} \mathbf{1}_{I}
$$

where $\widetilde{J}$ is the "parent" of $J$. Since $\left|x_{\widetilde{J}}\right| \leq\|x\|_{\dot{\mathbf{g}}_{\infty}^{q^{\prime}}}$ and

$$
f_{J} \sum_{I \in \mathcal{L}: I \subset J}\left|x_{I}\right|^{q^{\prime}} \mathbf{1}_{I} \leq\|x\|_{\dot{\mathbf{g}}_{\infty}^{q^{\prime}}}^{q^{\prime}}
$$


we conclude that

$$
f_{J}\left(\sum_{I \in \mathcal{L}: I \subset J}\left|\left(\mathrm{Av}^{*} x\right)_{I}\right|^{q^{\prime}} \mathbf{1}_{I}\right) d x \leq 2\|x\|_{\dot{\mathbf{g}}_{\infty}^{q^{\prime}}}^{q^{\prime}},
$$

which proves that $\mathrm{Av}^{*}$ is bounded. Therefore Av is a bounded operator in $\mathbf{g}_{1}^{q}$, which proves (4.2).

Statement (ii) follows from (4.2) by duality. Namely, take $g \in H^{p^{\prime}}, 1 / p+1 / p^{\prime}=1$, $\|g\|_{H^{p^{\prime}}} \leq 1$ and estimate

$$
\begin{aligned}
\left|\int_{\mathbb{X}} f g d x\right| & \leq \sum_{I \in \mathcal{L}} \int_{I}\left|\Delta_{I} f \Delta_{I} g\right| d x \leq \sum_{I \in \mathcal{L}} \int_{I}\left(\mathbb{E}_{I}\left|\Delta_{I} f\right|^{p}\right)^{1 / p}\left(\mathbb{E}_{I}\left|\Delta_{I} g\right|^{p^{\prime}}\right)^{1 / p^{\prime}} d x \\
& =\int \sum_{I \in \mathcal{L}}\left(\mathbb{E}_{I}\left|\Delta_{I} f\right|^{p}\right)^{1 / p}\left(\mathbb{E}_{I}\left|\Delta_{I} g\right|^{p^{\prime}}\right)^{1 / p^{\prime}} d x \\
& \leq \int\left(\sum_{I \in \mathcal{L}}\left(\mathbb{E}_{I}\left|\Delta_{I} f\right|^{p}\right)^{2 / p}\right)^{1 / 2}\left(\sum_{I \in \mathcal{L}}\left(\mathbb{E}_{I}\left|\Delta_{I} g\right|^{p^{\prime}}\right)^{2 / p^{\prime}}\right)^{1 / 2} \\
& \leq\left\|\left(\sum_{I \in \mathcal{L}}\left(\mathbb{E}_{I}\left|\Delta_{I} f\right|^{p}\right)^{2 / p}\right)^{1 / 2}\right\|\left\|_{p}\right\|\left(\sum_{I \in \mathcal{L}}\left(\mathbb{E}_{I}\left|\Delta_{I} g\right|^{p^{\prime}}\right)^{2 / p^{\prime}}\right)^{1 / 2} \|_{p^{\prime}} .
\end{aligned}
$$

By (4.2),

$$
\left\|\left(\sum_{I \in \mathcal{L}}\left(\mathbb{E}_{I}\left|\Delta_{I} g\right|^{p^{\prime}}\right)^{2 / p^{\prime}} \mathbf{1}_{I}\right)^{1 / 2}\right\|_{p^{\prime}} \leq C\|g\|_{H^{p^{\prime}}} .
$$

So by taking the supremum over $g \in H^{p^{\prime}},\|g\|_{H^{p^{\prime}}} \leq 1$, and taking into account that the dual of $H^{p}$ is isomorphic to $H^{p^{\prime}}$, we get

$$
\|f\|_{H^{p}} \leq C\left\|\left(\sum_{I \in \mathcal{L}}\left(\mathbb{E}_{I}\left|\Delta_{I} f\right|^{p}\right)^{2 / p}\right)^{1 / 2}\right\|_{p},
$$

which is exactly condition (ii).

4.1.2. Counterexamples in Theorem 4.1. To prove (iii), take $I_{0}=[0,2)$. Fix $n \in \mathbb{N}, n>2$ and let

$$
I_{k}=\left[0, r^{k}\right), \quad J_{k}=\left[r^{k}, r^{k-1}\right),
$$

where $r=1-1 / n, k=1,2, \ldots n$. Note that $I_{k-1}$ is a disjoint union of $I_{k}$ and $J_{k}$.

We assume here that $I_{k}, J_{k} \in \mathcal{L}_{k}$; we will only consider functions whose only nonzero martingale differences are $\Delta_{I_{k-1}} f, k=1,2, \ldots, n$, so the other intervals in $\mathcal{L}_{k}$ are irrelevant for our construction.

For $k=1,2, \ldots, n$ define

$$
\Delta_{I_{k-1}} f=\mathbf{1}_{J_{k}}-\alpha \mathbf{1}_{I_{k}}
$$

where $\alpha=1 /(n-1)$, so $\int_{\mathbb{X}} \Delta_{I_{k-1}} f d x=0$. Since $I_{k} \cap J_{k}=\varnothing$,

$$
\left|\Delta_{I_{k-1}} f\right|^{2}=\mathbf{1}_{J_{k}}+\alpha^{2} \mathbf{1}_{I_{k}} \leq \mathbf{1}_{J_{k}}+\alpha^{2} \mathbf{1}_{I_{1}} .
$$


Since the intervals $J_{k}, k=1,2, \ldots, n$, are disjoint, we can see that on $I_{0}$

$$
\left(\sum_{k=0}^{n-1}\left|\Delta_{I_{k}} f\right|^{2}\right)^{1 / 2} \leq\left(1+\sum_{k=1}^{n} \alpha^{2}\right)^{1 / 2} \leq\left(1+n \frac{1}{(n-1)^{2}}\right)^{1 / 2} \leq 2^{1 / 2}
$$

(each point $x \in I_{0}$ belongs to at most one of the intervals $J_{k}$, which contributes 1 to the sum, and each $I_{k}$ contributes $\alpha^{2}$ ). Therefore

$$
\left\|\left(\sum_{k=0}^{n-1}\left|\Delta_{I_{k}} f\right|^{2}\right)^{1 / 2}\right\|_{p} \leq 2^{1 / 2}
$$

On the other hand, for $x \in I_{k-1}$,

$$
\mathbb{E}_{I_{k-1}}\left|\Delta_{I_{k-1}} f\right|^{p}=\frac{1}{n}+(1-1 / n) \alpha^{p} \geq \frac{1}{n},
$$

so for $x \in I_{n}$,

$$
\sum_{k=1}^{n}\left(\mathbb{E}_{I_{k-1}}\left|\Delta_{I_{k-1}} f\right|^{p}\right)^{2 / p} \geq n\left(\frac{1}{n}\right)^{2 / p}=n^{1-2 / p} .
$$

Since $p>2$ we have $n^{1-2 / p} \rightarrow \infty$ as $n \rightarrow \infty$, so by increasing $n$ we can make the left side of (4.2) as large as we want (because $\left|I_{n}\right|=(1-1 / n)^{n}>1 / 2 e$ for sufficiently large $n$ ). By (4.4) the right side of (4.2) is uniformly bounded. Thus, the uniform (in all lattices) estimate (4.2) fails.

Repeating the construction (with $n \rightarrow \infty$ ) on disjoint intervals, we get a lattice where the uniform (in $f$ ) estimate (4.2) fails. But from this one can easily construct a function such that the right side of (4.2) is finite, but the left side is infinite.

The same construction allows us to prove statement (iv) as well. We can easily see that on $J=\cup_{k=1}^{n} J_{k}$,

$$
\left(\sum_{k=0}^{n-1}\left|\Delta_{I_{k}} f\right|^{2}\right)^{1 / 2} \geq 1
$$

Note that $|J|=1-r^{n}=1-(1 / n)^{n}$, so for sufficiently large $n$, we can estimate that $|J|>1 / 2$. Therefore

$$
\left\|\left(\sum_{k=0}^{n-1}\left|\Delta_{I_{k}} f\right|^{2}\right)^{1 / 2}\right\|_{p} \geq 2^{-1 / p}
$$

On the other hand, for $x \in I_{k-1}$,

$$
\mathbb{E}_{I_{k-1}}\left|\Delta_{I_{k-1}} f\right|^{p}=\frac{1}{n}+(1-1 / n) \alpha^{p}=\frac{1}{n}+\left(\frac{n-1}{n}\right)\left(\frac{1}{n-1}\right)^{p} \leq \frac{2}{n},
$$

so for $x \in I_{0}$,

$$
\sum_{k=1}^{n}\left(\mathbb{E}_{I_{k-1}}\left|\Delta_{I_{k-1}} f\right|^{p}\right)^{2 / p} \leq n\left(\frac{2}{n}\right)^{2 / p}=2^{2 / p} n^{1-2 / p}
$$


Therefore

$$
\left\|\left(\sum_{k=0}^{n-1}\left(\mathbb{E}_{I_{k}}\left|\Delta_{I_{k}} f\right|^{p}\right)^{2 / p}\right)^{1 / 2}\right\|_{p} \leq 2^{1 / p} n^{1 / 2-1 / p} \rightarrow 0 \quad \text { as } n \rightarrow \infty,
$$

because $p<2$. So for $p \in[1,2)$ the uniform estimate (4.3) fails, and from this it is easy to get a function for which the right side is finite, but the left side is infinite.

\subsubsection{Not a strong unconditional basis.}

Proposition 4.2. There exist a lattice $\mathcal{L}$ such that the martingale difference spaces $D_{I}$ do not form a strong unconditional basis.

This proposition also demonstrates that, unlike the $p=2$ case, the uniform boundedness in $L^{p}$ of the blocks $T_{I}$ of a martingale transform $T$ does not imply the boundedness of $T$ in $L^{p}$ if $p \neq 2$.

The proof of the proposition can be obtained by modifying the construction in Section 4.1.2. Define $I_{0}=[0,1)$. Fix $n \in \mathbb{N}, n>2$. Let split $I_{0}$ into two subintervals, $I_{1}$ and $J_{1}$, where $\left|J_{1}\right|=(1 / n)\left|I_{0}\right|$, so $\left|I_{1}\right|=(1-1 / n)\left|I_{0}\right|$, and let us split the intervals $I_{1}$ and $J_{1}$ into two equal subintervals, $I_{1}^{k}, J_{1}^{k}, k=1,2$. These four intervals will be children of $I_{0}$.

For an interval $I$, let $h_{I}$ be the Haar function (normalized in $L^{\infty}$ ),

$$
h_{I}=\mathbf{1}_{I_{+}}-\mathbf{1}_{I_{-}},
$$

where $I_{+}$and $I_{-}$are the right and left halves of $I$ respectively.

Define the martingale differences in $D_{I_{0}}$,

$$
\Delta_{I_{0}} f=h_{J_{1}}+\alpha h_{I_{1}}, \quad \Delta_{I_{0}} g=\beta\left(h_{J_{1}}+h_{I_{1}}\right),
$$

where, as in Section 4.1.2, $\alpha=(n-1)^{-1}$, and $\beta=n^{-p}\left(1+(n-1)^{1-p}\right)^{1 / p}$, so

$$
\left\|\Delta_{I_{0}} f\right\|_{p}=\left\|\Delta_{I_{0}} g\right\|_{p}
$$

We then apply the same construction to the "children" $I_{1}^{k}$ of $I_{0}$, then to all "children" $I_{2}^{j}$ of all $I_{1}^{k}$ and so on. Note, that we do not care about the "children" of the "smaller" intervals $J_{r}^{k}$, because we set the martingale differences to zero for all intervals different from one of $I_{k}^{j}$.

So, we get the collection of intervals $I_{k}^{j}$ and the corresponding martingale differences $\Delta_{I_{k}^{j}} f$ and $\Delta_{I_{k}^{j}} g$, constructed in the same manner as in (4.5).

Now let us notice that this construction just models the construction from Section 4.1.2. Namely, for every $k \in \mathbb{N}$, the total length of the intervals $I_{k}^{j}$ is exactly the length of the interval $I_{k}$ from Section 4.1.2. It is easy to see that the function $\sum_{j}\left|\Delta_{I_{k}^{j}} f\right|$ and the function $\left|\Delta_{I_{k}} f\right|$ from Section 4.1.2 have the same distribution function. 
Moreover, the corresponding square functions

$$
\left(\sum_{k, j}\left|\Delta_{I_{k}^{j}} f\right|^{2}\right)^{1 / 2} \text { and }\left(\sum_{k}\left|\Delta_{I_{k}} f\right|^{2}\right)^{1 / 2}
$$

also have the same distribution function.

The distribution functions of the square function

$$
\left(\sum_{k, j}\left|\Delta_{I_{k}^{j}} g\right|^{2}\right)^{1 / 2}
$$

and of the function

$$
\left(\sum_{k}\left(\mathbb{E}_{I_{k}}\left|\Delta_{I_{k}}\right|^{p}\right)^{2 / p}\right)^{1 / 2}
$$

from Section 4.1.2 also coincide. Therefore, all estimates from Section 4.1.2 apply here, and repeating the reasoning from that section we prove the proposition.

\section{2. "Paraproduct" version of the embedding theorem}

Let $b=\left\{b^{I}\right\}_{I \in \mathcal{C}}$ be a family of functions such that $b^{I}$ is supported on $I$ and is constant on "children" of $I$. Define a "paraproduct type" operator $\tilde{\pi}=\widetilde{\pi}_{b}$ by

$$
\tilde{\pi}_{b} f(\cdot, k)=\sum_{I \in \mathcal{L}: \mathrm{rk}(I)=k}\langle f\rangle_{I} b^{I} .
$$

If $b^{I}=\Delta_{I} b$ for some scalar function $b$, this is just the vector representation of the classical paraproduct. However, here we do not assume that the $b^{I}$ are orthogonal to constants, so here $b$ is simply a collection of functions $b^{I}$.

We want to know when this operator is a bounded operator $L^{p} \rightarrow L^{p}\left(\ell^{q}\right)$, $1<p<\infty$ (or from $\widetilde{\mathcal{H}}^{p} \rightarrow L^{p}\left(\ell^{q}\right)$, if we are also interested in the case $p=1$ ).

If each $b^{I}$ is constant on $I$, the answer is given by Theorem 2.7 above, and it does not depend on $p \in[1, \infty)$. In the general case, if we do not assume that the lattice is homogeneous, the answer generally depends on $p$; one can easily come up with a counterexample in the simplest situation when only the $b^{I}$ with $I$ in a disjoint family are nonzero.

Theorem 4.3. Let $p \in[1, \infty), q \in(1, \infty)$. The operator $\tilde{\pi}_{b}$ defined above is a bounded operator $\widetilde{\mathcal{H}}^{p} \rightarrow L^{p}\left(\ell^{q}\right)$ if and only if

$$
\sup _{I \in \mathcal{L}} f_{I}\left(\sum_{J \in \mathcal{L}: J \subset I}\left|b^{J}(x)\right|^{q}\right)^{\frac{1}{q} p} d x=K^{p}<\infty
$$

Moreover, the norm of $\tilde{\pi}_{b}$ is bounded by $C K$, where $C=C(p)$.

Proof. (Necessity and the easy case $p \leq q$ ). The necessity of the condition (4.7) is trivial, one just needs to test the boundedness of $\tilde{\pi}_{b}$ on the characteristic function $\mathbf{1}_{I}, I \in \mathcal{L}$, and when computing the $L^{p}\left(\ell^{q}\right)$-norm count only the $\langle f\rangle_{J} b^{J}$ corresponding to $J \subset I$. 
To be completely honest, this test function works fine for $p>1$; for $p=1$ one should take for the test function $f=\mathbf{1}_{I}-\alpha \mathbf{1}_{K \backslash I}$, where $K$ is the first ancestor of $I$ for which $2|I| \leq|K|$, and $\alpha \in \mathbb{R}$ is such, that $\int f d x=0$ (if no such $K$ exists, one can take $\mathbf{1}_{I}$ for the test function). We refer the reader to the proof of Theorem 2.7 for the details.

To prove the sufficiency, let us first fix the notation. As in the proof of Theorem 2.7, let $E_{k}:=\left\{x \in \mathbb{X}: M_{\mathcal{L}} f(x)>2^{k}\right\}$ and let $\mathcal{E}_{k}:=\left\{I \in \mathcal{L}: I \subset E_{k}\right\}$.

For $\mathcal{E} \subset \mathcal{L}$ let $\left(\tilde{\pi}_{b} f\right)_{\mathcal{E}}$ be the coordinate projection of $\widetilde{\pi}_{b} f$ corresponding to the set $\mathcal{E}$, meaning that the sum in (4.6) is taken only over $I \in \mathcal{E}$.

The sufficiency for $p \leq q$ is proved in exactly the same way as in Theorem 2.7: using the same reasoning as was used there, we get the analogue of (2.16), namely that

$$
\sum_{k \in \mathbb{Z}}\left\|\left(\widetilde{\pi}_{b} f\right)_{\mathcal{E}_{k} \backslash \mathcal{E}_{k+1}}\right\|_{L^{p}\left(\ell^{q}\right)}^{p} \leq C\|f\|_{p}^{p},
$$

(this inequality holds for all $p, q \in(1, \infty)$ with $C=C(p, q)$ ).

If $p \leq q$, this gives immediately the desired estimate in the same way as in the proof of Theorem 2.7. We did not use the fact that the $\alpha_{I}$ were constants there; the estimate works for arbitrary functions.

4.2.1. Stopping moments and the hard estimate in Theorem 4.3. To treat the estimate in the situation when $p>q$ we employ the stopping moment technique.

First of all let us note that if $p>q>1$, then $p>1$, so the space $\widetilde{\mathcal{H}}^{p}$ is isomorphic to $L^{p}$. This means that without loss of generality we can assume $f \geq 0$, which we will do in what follows (note that we cannot do this for $\widetilde{\mathcal{H}}^{1}$ ).

So, let us assume $f \geq 0, f \in L^{p}$.

To prove the theorem we are going to construct the generations $\mathcal{G}_{k}^{*}$ of stopping intervals. For an interval $J \in \mathcal{L}$ let $\mathcal{G}^{*}(J)$ be the collection of the maximal (with respect to inclusion) intervals $I \in \mathcal{L}, I \subset J$ such that

$$
\langle f\rangle_{I}>2\langle f\rangle_{J}
$$

Fix some $k_{0} \in \mathbb{Z}$ (later we let $k_{0} \rightarrow-\infty$ ) and define $\mathcal{G}_{1}^{*}:=\mathcal{L}_{k_{0}}$,

$$
\mathcal{G}_{k+1}^{*}:=\bigcup_{I \in \mathcal{G}_{k}^{*}} \mathcal{G}^{*}(I), \quad \mathcal{G}^{*}=\bigcup_{k \geq 1} \mathcal{G}_{k}^{*}
$$

For an interval $J$ let $\mathcal{L}(J):=\{I \in \mathcal{L}: I \subset J\}$, and let

$$
\mathcal{F}(J):=\mathcal{L}(J) \backslash \cup_{I \in \mathcal{G}^{*}(J)} \mathcal{L}(I) .
$$

We will need the following simple lemma.

Lemma 4.4. Let $J \in \mathcal{L}$. Then,

(i) for any $I \in \mathcal{F}(J)$ we have $\langle f\rangle_{I} \leq 2\langle f\rangle_{J}$;

(ii) $\sum_{I \in \mathcal{G}^{*}(J)}|I| \leq|J| / 2$.

Proof. The proof is an easy exercise for the reader. 
Corollary 4.5. The collection $\mathcal{G}^{*}$ of stopping intervals satisfies the Carleson measure condition

$$
\sum_{I \in \mathcal{G}^{*}, I \subset J}|I| \leq 2|J| \quad \forall J \in \mathcal{L}
$$

Proof. For an interval $I \in \mathcal{L}$ let $\mathcal{G}(I)$ be the collection of intervals which are maximal for the family $\left\{I^{\prime} \in \mathcal{G}^{*}: I^{\prime} \subset I\right\}$. Denote $\mathcal{G}_{1}:=\mathcal{G}(J)$, and for $k \geq 1$ define

$$
\mathcal{G}_{k+1}:=\bigcup_{I \in \mathcal{G}_{k}} \mathcal{G}^{*}(I)=\bigcup_{I \in \mathcal{G}_{k}} \mathcal{G}(I) .
$$

The collection $\mathcal{G}_{1}=\mathcal{G}(J)$ consists of disjoint intervals, so $\sum_{I \in \mathcal{G}_{1}} \leq|J|$. Statement (ii) of the lemma implies that

$$
\sum_{I \in \mathcal{G}_{k+1}}|I| \leq \frac{1}{2} \sum_{I \in \mathcal{G}_{k}}|I|, \quad k \geq 1
$$

Since $\left\{I \in \mathcal{G}^{*}: I \subset J\right\}=\bigcup_{k=1}^{\infty} \mathcal{G}_{k}$, we immediately get (4.9) by summing a geometric series.

Remark 4.6. The estimate (4.9) means that the sequence $w=\left\{w_{I}\right\}_{I \in \mathcal{L}}$

$$
w_{I}= \begin{cases}|I|^{1 / p}, & I \in \mathcal{G}^{*}, \\ 0, & I \notin \mathcal{G}^{*},\end{cases}
$$

belongs to the space $\mathbf{g}_{\infty}^{p}$.

Recall that for $\mathcal{E} \subset \mathcal{L}$ the coordinate projection $\left(\tilde{\pi}_{b} f\right)_{\mathcal{E}}$ is defined by taking the sum in (4.6) only over $I \in \mathcal{E}$.

Let $\mathcal{L}^{k_{0}}:=\bigcup_{k \geq k_{0}} \mathcal{L}_{k}$. To prove the theorem it is sufficient to get an estimate of the norm of $\left(\widetilde{\pi}_{b} f\right)_{\mathcal{L}^{k_{0}}}$ uniform in $k_{0}$. Since $\mathcal{L}^{k_{0}}=\bigcup_{J \in \mathcal{G}^{*}} \mathcal{F}(J)$ (see (4.8) for the definition of $\mathcal{F}(J)$ ), we have the decomposition

$$
\left(\tilde{\pi}_{b} f\right)_{\mathcal{L}^{k_{0}}}=\sum_{J \in \mathcal{G}^{*}}\left(\widetilde{\pi}_{b} f\right)_{\mathcal{F}(J)} .
$$

Statement (i) of Lemma 4.4 together with the assumption (4.7) imply that

$$
\left\|\left(\tilde{\pi}_{b} f\right)_{\mathcal{F}(J)}\right\|_{L^{p}\left(\ell^{q}\right)} \leq 2 K\langle f\rangle_{J}|J|^{1 / p} .
$$

Take $g \in L^{p^{\prime}},\|g\|_{p^{\prime}} \leq 1$. Then

$$
\int_{\mathbb{X}}\left\|\left(\widetilde{\pi}_{b} f\right)_{\mathcal{L}^{k_{0}}}(x, \cdot)\right\|_{\ell^{q}}|g(x)| d x \leq \sum_{J \in \mathcal{G}^{*}} \int_{J}\left\|\left(\widetilde{\pi}_{b} f\right)_{\mathcal{F}(J)}(x, \cdot)\right\|_{\ell^{q}}|g(x)| d x
$$

Let us denote by $G(J)$ the "shadow' of $\mathcal{G}^{*}(J), G(J):=\cup_{I \in \mathcal{G}^{*}(J)} I$. Each integral in the sum on the right-hand side of (4.11) can be split as

$$
\int_{J} \cdots=\int_{J \backslash G(J)} \cdots+\int_{G(J)} \cdots=A(J)+B(J) .
$$


The sum of $A(J)$ is easy to estimate. Namely, using (4.10) and the fact that the sets $J \backslash G(J), J \in \mathcal{G}^{*}$ are disjoint we can write

$$
\begin{aligned}
\sum_{J \in \mathcal{G}} A(J) & \leq \sum_{J \in \mathcal{G}^{*}}\left\|\left(\widetilde{\pi}_{b} f\right)_{\mathcal{F}(J)}\right\|_{L^{p}\left(\ell^{q}\right)}\left\|g \mathbf{1}_{J \backslash G(J)}\right\|_{p^{\prime}} \\
& \leq\left(\sum_{J \in \mathcal{G}^{*}}\left\|\left(\widetilde{\pi}_{b} f\right)_{\mathcal{F}(J)}\right\|_{L^{p}\left(\ell^{q}\right)}^{p}\right)^{1 / p}\left(\sum_{J \in \mathcal{G}^{*}}\left\|g \mathbf{1}_{J \backslash G(J)}\right\|_{p^{\prime}}^{p^{\prime}}\right)^{1 / p^{\prime}} \quad \text { Hölder } \\
& \leq\left(\sum_{J \in \mathcal{G}^{*}}\left\|\left(\widetilde{\pi}_{b} f\right)_{\mathcal{F}(J)}\right\|_{L^{p}\left(\ell^{q}\right)}^{p}\right)^{1 / p}\|g\|_{p^{\prime}} \quad J \backslash G(J) \text { are disjoint } \\
& \leq 2 K\left(\sum_{J \in \mathcal{G}^{*}}\left|\langle f\rangle_{J}\right|^{p}|J|\right)^{1 / p}\|g\|_{p^{\prime}} \quad \text { by (4.10). }
\end{aligned}
$$

The collection $\mathcal{G}^{*}$ satisfies the Carleson measure condition (4.9), so by the Carleson embedding theorem (Theorem 2.7 for $p=q$; see Remark 4.6) we get that

$$
\sum_{J \in \mathcal{G}^{*}}\left|\langle f\rangle_{J}\right|^{p}|J| \leq C\|f\|_{p}^{p}
$$

which gives the desired estimate for $\sum_{J \in \mathcal{G}^{*}} A(J)$.

To estimate the sum of the $B(J)$, let us notice that for $J \in \mathcal{G}^{*}$ the function $\left(\widetilde{\pi}_{b} f\right)_{\mathcal{F}(J)}$ is constant on intervals $I \in \mathcal{G}^{*}(J)$, so the integral $B(J)$ does not change if we replace $g$ there by the function $g_{J}$,

$$
g_{J}:=\sum_{I \in \mathcal{G}^{*}(J)}\langle g\rangle_{I} \mathbf{1}_{I}
$$

Since

$$
\left\|g_{J}\right\|_{p^{\prime}}^{p^{\prime}}=\sum_{I \in \mathcal{G}_{k+1}^{*}, I \subset J}\left|\langle g\rangle_{I}\right|^{p^{\prime}}|I|,
$$

using (4.10) we can estimate

$$
B(J) \leq 2 K\langle f\rangle_{J}|J|^{1 / p}\left(\sum_{I \in \mathcal{G}^{*}(J)}\left|\langle g\rangle_{I}\right|^{p^{\prime}}|I|\right)^{1 / p^{\prime}} .
$$

Since the collections $\mathcal{G}^{*}(J), J \in \mathcal{G}^{*}$ are disjoint and $\bigcup_{J \in \mathcal{G}^{*}} \mathcal{G}^{*}(J) \subset \mathcal{G}^{*}$, applying the Hölder inequality to $(4.13)$ we get

$$
\sum_{J \in \mathcal{G}^{*}} B(J) \leq\left(\sum_{J \in \mathcal{G}^{*}}\left|\langle f\rangle_{J}\right|^{p}|J|\right)^{1 / p}\left(\sum_{I \in \mathcal{G}^{*}}\left|\langle g\rangle_{I}\right|^{p^{\prime}}|I|\right)^{1 / p^{\prime}} .
$$

The first factor on the right side was already bounded by $C\|f\|_{p}$ in (4.12). The second factor is estimated in exactly the same way by $\|g\|_{p^{\prime}}$. Note that while the collection $\mathcal{G}^{*}$ of stopping intervals depends on $f$, only the Carleson measure condition (4.9) was used to prove (4.12), so (4.12) holds for $g$ (with $p^{\prime}$ instead of $p$ ). 
Combining (4.14) with the estimate for the sum of $A(J) \mathrm{s}$, we get

$$
\int_{E_{k_{0}}}\left\|\left(\widetilde{\pi}_{b} f\right)_{\mathcal{E}_{k_{0}}}(x, \cdot)\right\|_{\ell^{q}}|g(x)| d x \leq C\|f\|_{p}\|g\|_{p^{\prime}}
$$

Letting $k_{0} \rightarrow-\infty$ concludes the proof.

Remark. The estimates (4.10) and (4.12) imply that, for $p>1$,

$$
\sum_{J \in \mathcal{G}^{*}}\left\|\left(\widetilde{\pi}_{b} f\right)_{\mathcal{F}(J)}\right\|_{L^{p}\left(\ell^{q}\right)}^{p} \leq C\|f\|_{p}^{p}
$$

Since $\|x\|_{\ell^{q}} \leq\|x\|_{\ell^{p}}$ for $p \leq q$, the conclusion of Theorem 4.3 for $1<p \leq q$ follows easily from (4.15).

This reasoning does not work for $p=1$ : if $p=1$ we cannot assume without loss of generality that $f \geq 0$, and this assumption was essential in the construction.

The proof we presented earlier for the easy case $p \leq q$ works for all $p \geq 1$.

\subsection{Bounds for paraproducts}

We will need the following simple lemma.

Lemma 4.7. Let $I$ be a disjoint union of sets $I_{1}$ and $I_{2}$, and let $h$ be a "Haar function", i.e., $h=\alpha_{1} \mathbf{1}_{I_{1}}+\alpha_{2} \mathbf{1}_{I_{2}}$ and $\int_{\mathbb{X}} h d x=0$. Then, assuming without loss of generality that $\left|I_{1}\right| \leq\left|I_{2}\right|$ we get that for $p \in[1, \infty)$

$$
\|h\|_{p}^{p} \leq 2\left\|h \mathbf{1}_{I_{1}}\right\|_{p}^{p}
$$

and that the inverse Hölder inequality holds:

$$
\|h\|_{p}\|h\|_{p^{\prime}} \leq 2\|h\|_{2}^{2}, \quad 1 / p+1 / p^{\prime}=1 .
$$

Proof of Lemma 4.7. Assume without loss of generality that $\left|I_{1}\right| \leq\left|I_{2}\right|$. The condition $\int_{\mathbb{X}} h d x=0$ means that

$$
\alpha_{1}\left|I_{1}\right|=-\alpha_{2}\left|I_{2}\right|
$$

which immediately implies $\left|\alpha_{2}\right| \leq\left|\alpha_{1}\right|$. Then

$$
\int_{\mathbb{X}}|h|^{p} d x=\left|\alpha_{1}\right|^{p}\left|I_{1}\right|+\left|\alpha_{2}\right|^{p}\left|I_{2}\right|=\left|\alpha_{1}\right|^{p}\left|I_{1}\right|+\left|\alpha_{2}\right|^{p-1}\left|\alpha_{1}\right|\left|I_{1}\right| \leq 2\left|\alpha_{1}\right|^{p}\left|I_{1}\right|,
$$

so

$$
\|h\|_{p}^{p} \leq 2\left\|h \mathbf{1}_{I}\right\|_{p}^{p}
$$

and similarly for $p^{\prime}$.

Since for constant functions the Hölder inequality becomes an identity, using the above estimate we can write

$$
\|h\|_{p}\|h\|_{p^{\prime}} \leq 2\left\|h \mathbf{1}_{I}\right\|_{p}\left\|h \mathbf{1}_{I}\right\|_{p^{\prime}}=2\left\|h \mathbf{1}_{I}\right\|_{2}^{2} \leq 2\|h\|_{2}^{2} .
$$


Theorem 4.8. Let $b=\left\{\Delta_{I} b\right\}_{I \in \mathcal{L}}$ be a martingale difference sequence, and let $p \in[1, \infty), q \in(1, \infty)$. Then:

(i) The paraproduct $\pi_{b}$ is a bounded operator from $\widetilde{\mathcal{H}}^{p}$ to $\mathcal{H}_{q}^{p}$ if and only if

$$
\sup _{I \in \mathcal{L}} f_{I}\left(\sum_{J \in \mathcal{L}: J \subset I}\left|\Delta_{J} b(x)\right|^{q}\right)^{\frac{1}{q} p} d x=: K^{p}<\infty .
$$

Moreover,

$$
K \leq\left\|\pi_{b}\right\|_{\widetilde{\mathcal{H}}^{p} \rightarrow \mathcal{H}_{q}^{p}} \leq C K
$$

where $C=C(p, q)$.

(ii) The paraproduct $\pi_{b}^{(*)}$ is a bounded operator in $\widetilde{\mathcal{H}}^{p}=\widetilde{\mathcal{H}}_{2}^{p}$ if and only if $b \in$ $\mathcal{B M O}=\mathrm{BMO}_{2}$. Moreover,

$$
\frac{1}{C}\|b\|_{\mathcal{B M O}} \leq\left\|\pi_{b}^{(*)}\right\|_{\widetilde{\mathcal{H}}^{p} \rightarrow \widetilde{\mathcal{H}}^{p}} \leq C\|b\|_{\mathcal{B M O}}
$$

where $C=C(p)$.

Remark 4.9. For $q=2$, statement (i) of the theorem describes the boundedness of the paraproduct $\pi_{b}$ in $\widetilde{\mathcal{H}}^{p}$ (or equivalently, in $\widetilde{H}^{p}$ ). For $p \in(1, \infty)$ this is equivalent to the boundedness of $\pi_{b}$ in $L^{p}$.

Note that, unlike the condition $b \in \mathcal{B M O}$, which is necessary and sufficient for the boundedness of $\pi_{b}^{(*)}$ in all $\widetilde{\mathcal{H}}^{p}$, the above condition (4.16) (for $q=2$, for example) does depend on $p$.

Remark 4.10. Note that the condition (4.16) (for $p=q=2$ ) is weaker then the condition $b \in$ BMO. Since by Proposition 1.5

$$
M_{b}=\pi_{b}+\pi_{b}^{*}+\Lambda_{b}+R_{b},
$$

and $\lambda_{b}$ and $R_{b}$ commute with all martingale multipliers, the above theorem implies, in particular, that unlike the homogeneous case, it is impossible in general to characterize $b \in \mathrm{BMO}$ via boundedness of the commutators of $M_{b}$ with martingale multipliers.

Proof of Theorem 4.8. The statement (i) is easy. The "only if" part and the estimate $K \leq\left\|\pi_{b}\right\|$ follow from testing the boundedness of $\pi_{b}$ on functions $\mathbf{1}_{I}, I \in \mathcal{L}$. The "if" part with the estimate $\left\|\pi_{b}\right\| \leq C K$ follow from Theorem 4.3 above.

Let us prove statement (ii). Notice that, by Proposition 1.5,

$$
\pi_{b}^{(*)}=\pi_{b}^{*}+\Lambda_{b}^{1}
$$

If $b \in \mathcal{B M O}$, we know that, for any $p^{\prime} \in(1, \infty)$,

$$
\sup _{I \in \mathcal{L}} f_{I}\left(\sum_{J \in \mathcal{L}: J \subset I}\left|\Delta_{J} b(x)\right|^{2}\right)^{\frac{1}{2} p^{\prime}} d x \leq C\|b\|_{\mathcal{B} \mathcal{M O}}^{p^{\prime}}
$$

Taking $p^{\prime}$ to be the exponent dual to $p, 1 / p+1 p^{\prime}=1$, we get that, by (i), $\pi_{b}$ is bounded in $\widetilde{\mathcal{H}}^{p^{\prime}}$, so by duality $\pi_{b}^{*}$ is bounded in $\widetilde{\mathcal{H}}^{p}$. 
Since, by Proposition 1.5,

$$
\Lambda_{b}^{1} f=\sum_{I \in \mathcal{L}} \Delta_{I}\left[\left(\Delta_{I} b\right)\left(\Delta_{I} f\right)\right]=\sum_{I \in \mathcal{L}}\left(\Delta_{I} b\right)\left(\Delta_{I} f\right)-\sum_{I \in \mathcal{L}} \mathbb{E}_{I}\left(\left(\Delta_{I} b\right)\left(\Delta_{I} f\right)\right),
$$

and by the definition of $\mathcal{B M O}$ we have $\left\|\Delta_{I} b\right\|_{\infty} \leq\|b\|_{\mathcal{B M O}}$, we can conclude that $\Lambda_{b}^{1}$ is bounded in $\widetilde{\mathcal{H}}^{p}$. Indeed, since $\left\|\Delta_{I} b\right\|_{\infty} \leq\|b\|_{\mathcal{B M O}}$,

$$
\int_{\mathbb{X}}\left(\sum_{I \in \mathcal{L}}\left|\left(\Delta_{I} b\right)\left(\Delta_{I} f\right)\right|^{2}\right)^{p / 2} d x \leq\|b\|_{\mathcal{B} \mathcal{M O}}^{p} \int_{\mathbb{X}}\left(\sum_{I \in \mathcal{L}}\left|\Delta_{I} f\right|^{2}\right)^{p / 2} d x=\|b\|_{\mathcal{B} \mathcal{M O}}^{p}\|f\|_{\widetilde{\mathcal{H}}^{p}}^{p} .
$$

By the Fefferman-Stein maximal theorem we get from this inequality

$$
\int_{\mathbb{X}}\left(\sum_{I \in \mathcal{L}}\left|\mathbb{E}_{I}\left(\left(\Delta_{I} b\right)\left(\Delta_{I} f\right)\right)\right|^{2}\right)^{p / 2} d x \leq C\|b\|_{\mathcal{B} \mathcal{M O} \mathcal{O}}^{p}\|f\|_{\widetilde{\mathcal{H}}^{p}}^{p}
$$

Hence both sums in (4.17) can be controlled and we get that $\Lambda_{b}^{1}$ is bounded in $\widetilde{\mathcal{H}}^{p}$.

Assume now that $\pi_{b}^{(*)}$ is bounded in $\widetilde{\mathcal{H}}^{p}$, so that

$$
\left(\pi_{b}^{(*)}\right)^{*}=\pi_{b}+\left(\Lambda_{b}^{1}\right)^{*}=\pi_{b}+\Lambda_{b}^{1}
$$

is bounded in $\widetilde{\mathcal{H}}^{p^{\prime}}$. Testing this operator on the functions $\mathbf{1}_{I}$ and counting in the result only martingale differences with $J \subset I$, we get

$$
\sup _{I \in \mathcal{L}} f_{I}\left(\sum_{J \in \mathcal{L}: J \subset I}\left|\Delta_{J} b(x)\right|^{2}\right)^{\frac{1}{2} p^{\prime}} d x \leq K^{p^{\prime}}<\infty, \quad K=\left\|\left(\pi_{b}^{(*)}\right)^{*}\right\|_{\mathcal{H}^{p \rightarrow \mathcal{H}^{p}}}
$$

By (i) this means that $\pi_{b}$ is bounded in $\mathcal{H}^{p^{\prime}}$ (with norm at most $C K$ ), and so $\Lambda_{b}^{1}$ is also bounded in $\mathcal{H}^{p^{\prime}}$ with the norm at most $C_{1} K$. By duality, $\Lambda_{b}^{1}$ is bounded in $\mathcal{H}^{p}$ (with the same norm).

The estimate (4.18) also implies that $\left\|\Delta_{I} b\right\|_{p^{\prime}} \leq K|I|^{1 / p^{\prime}}$.

To prove that $b \in \mathcal{B M O}_{q^{\prime}}$ there remains only to show that, for all $I \in \mathcal{L}$,

$$
\left\|\Delta_{I} b\right\|_{\infty} \leq C K
$$

Assume that $\left\|\Delta_{I} b\right\|_{\infty} \geq 2^{1 / p} K$, because otherwise we already have the desired estimate. Let $J \in \operatorname{child}(I)$ be an interval where $\left\|\Delta_{I} b\right\|_{\infty}$ is attained. Then

$$
2 K^{p}|J| \leq\left\|\Delta_{I} b\right\|_{\infty}^{p}|J| \leq\left\|\Delta_{I} b\right\|_{p}^{p} K^{p}|I|,
$$

so $|J| \leq|I| / 2$.

Define a test function $h$ by

$$
h:=\mathbf{1}_{J}-\alpha \mathbf{1}_{I \backslash J}, \quad \alpha=|J| /(|I|-|J|) \leq 1,
$$

so $\int_{\mathbb{X}} h d x=0$. Since $|J| \leq|I \backslash J|$, Lemma 4.7 implies that

$$
\|h\|_{p}^{p} \leq 2\left\|\mathbf{1}_{J}\right\|_{p}^{p}=2|J| .
$$


Our test function $\Delta_{I} h=h$ is the only nonzero martingale difference, so (4.17) yields

$$
\left\|h \Delta_{I} b\right\|_{p} \leq\left\|\Lambda_{b}^{1} h\right\|_{p}+\left\|\mathbb{E}_{I}\left(h \Delta_{I} b\right)\right\|_{p}
$$

We can estimate

$$
\left\|h \Delta_{I} b\right\|_{p} \geq\left\|\mathbf{1}_{J} h \Delta_{I} b\right\|_{p}=|J|^{1 / p}\left\|\Delta_{I} b\right\|_{\infty} .
$$

On the other hand,

$$
\left\|\Lambda_{b}^{1} h\right\|_{p} \leq C_{1} K\|h\|_{p}=C_{1} K(2|J|)^{1 / p}
$$

and

$$
\begin{aligned}
\left\|\mathbb{E}_{I}\left(h \Delta_{I} b\right)\right\|_{p} & \leq|I|^{1 / p} f_{I}\left|h \Delta_{I} b\right| d x \leq|I|^{1 / p-1}\left\|\Delta_{I}\right\|_{p^{\prime}}\|h\|_{p} \\
& \leq 0|I|^{1 / p-1} K|I|^{1 / p^{\prime}}(2|J|)^{1 / p}=2^{1 / p} K|J|^{1 / p} .
\end{aligned}
$$

Combining the preceeding we get that

$$
|J|^{1 / p}\left\|\Delta_{I} b\right\|_{\infty} \leq 2^{1 / p} C_{1} K|J|^{1 / p}+2^{1 / p} K|J|^{1 / p},
$$

so $\left\|\Delta_{I} b\right\|_{\infty} \leq C K, C=2^{1 / p}\left(C_{1}+1\right)$.

\section{Boundedness of commutators in $L^{p}$}

\subsection{Sufficiency}

We start with a simple proposition.

Proposition 5.1. Let $p \in(1, \infty)$, and let $T$ be a martingale transform bounded in $L^{p}$ (equivalently in $\widetilde{H}_{2}^{p}$ ). Let $b$ be a locally integrable function.

If the formal sum $b_{0}:=\sum_{I \in \mathcal{L}} \Delta_{I} b$ is in $\mathcal{B M O}$, the commutator $\left[M_{b}, T\right]=$ $M_{b} T-T M_{b}$ is bounded in $L^{p}$ (equivalently in $\left.\widetilde{H}^{p}\right)$. Moreover, for $C=C(p)$,

$$
\left\|\left[M_{b}, T\right]\right\|_{L^{p} \rightarrow L^{p}} \leq C\|T\|_{L^{p} \rightarrow L^{p}}\left\|b_{0}\right\|_{\mathcal{B M O}}
$$

Remark. Note that the case $p=1$ is not included here. While the condition $b_{0} \in \mathcal{B M O}$ is necessary and sufficient for the boundedness of the paraproduct $\pi_{b}$ in $\widetilde{H}^{1}$, this condition is not sufficient for the boundedness of the adjoint $\pi_{b}^{*}$ there, even in the simplest case of the standard dyadic grid.

This can be seen easily by going to the dual space and noticing that the condition $b \in \mathrm{BMO}$ is not sufficient for the boundedness of the paraproduct $\pi_{b}$ in BMO (here we are considering the simplest case of the standard dyadic grid on $\mathbb{R}$, so all BMO spaces are the same). Since the condition $f \in$ BMO does not imply any bounds on the averages $\langle f\rangle_{I}$, one can take an unbounded function $f \in \mathrm{BMO}$ (so the averages $\langle f\rangle_{I}$ are not uniformly bounded) and easily construct a function $b \in \mathrm{BMO}$ such that $\pi_{b} f \notin \mathrm{BMO}$.

Proof of Proposition 5.1. By Proposition 1.5,

$$
M_{b}=\pi_{b}^{(*)}+\Lambda_{b}^{0}+\pi_{b}+R_{b} .
$$


The operator $\Lambda_{b}^{0}$ commutes with all martingale transforms, so we can exclude it from the commutator. Since $T R_{b}=R_{b} T=0$, we can exclude $R_{b}$ as well, so

$$
\left[M_{b}, T\right]=\left[\pi_{b}+\pi_{b}^{(*)}, T\right] .
$$

Therefore, if $\pi_{b}$ and $\pi_{b}^{(*)}$ are bounded, the commutator is bounded as well. But according to Theorem 4.8, the condition $b \in$ BMO implies the boundedness of both of the paraproducts $\pi_{b}$ and $\pi_{b}^{(*)}$ (for $q=2$ condition (i) of Theorem 4.8 follows from condition (ii) there).

It will be shown later that in the case where $\mathfrak{A}_{-\infty}^{0, \text { fin }} \cap \mathcal{L}=\varnothing$ and the martingale transform $T$ has the right "mixing" properties, the condition $\sum_{I \in \mathcal{L}} \Delta_{I} b \in \mathcal{B M O}$ is also necessary for the boundedness of the commutator. If $\mathfrak{A}_{-\infty}^{0, \text { fin }} \cap \mathcal{L} \neq \varnothing$, the sufficient condition $\sum_{I \in \mathcal{L}} \Delta_{I} b \in \mathcal{B} \mathcal{M O}$ can be relaxed a little. As will be shown below in Section 5.2, this relaxed condition is also necessary (again if the martingale transform $T$ has the right "mixing" properties).

\subsection{Necessity}

We want to state and prove an inverse (at least partial) to Proposition 5.1. Of course, to prove such a theorem one needs to make some additional assumptions about the martingale transform $T$ (for example, the identity is a martingale transform, and it commutes with everything).

Definition 5.2. Let $T$ be a martingale transform. Following S. Janson [8], we say that an interval $I \in \mathcal{L}$ with parent $I^{\prime}$ is $(p, \varepsilon, K)$ non-degenerate for $T$ if there exists $h=h_{I^{\prime}} \in D_{I^{\prime}}=\Delta_{I^{\prime}} L^{2}$, such that

(i) $\|h\|_{p}=1$,

(ii) $\left.h\right|_{I}=0$,

(iii) $\left\|\mathbf{1}_{I} T_{I^{\prime}} h\right\|_{p} \geq \varepsilon$,

(iv) $\|h\|_{\infty} \leq K\left|I^{\prime}\right|^{-1 / p}$ if $I$ is "small", namely if $|I|<\left|I^{\prime}\right| / K$.

The last condition (iv) means that for "small" intervals $I$ the function $h$ has to be "spread" over the interval $I^{\prime}$. If we omit condition (iv), we get the definition of a $(p, \varepsilon)$ non-degenerate interval.

We say that the martingale transform $T$ is weakly $(p, \varepsilon, K)$ mixing if each interval $I$ with a parent is either $(p, \varepsilon, K)$ non-degenerate for $T$ or $\left(p^{\prime}, \varepsilon, K\right)$ nondegenerate for the adjoint $T^{*}$.

We say that the martingale transform $T$ is strongly $(p, \varepsilon, K)$ mixing if each interval $I$ with a parent is $(p, \varepsilon, K)$ non-degenerate.

Using the notion of $(p, \varepsilon)$ non-degenerate intervals, one can define weakly and strongly $(p, \varepsilon)$ mixing martingale transforms.

Remark. The above definition of weakly $(p, \varepsilon)$ mixing martingale transform is essentially a restatement (and a generalization) of the definition of a non-degenerate 
transform from [8]. It was given there for the case of the uniform $r$-adic lattice, with all the operators $T_{I}$ being equal (after canonical identification of the subspaces $\left.D_{I}\right)$.

Recall, that in our notation the $r$-adic lattice can be represented as a union of generations $\mathcal{L}_{k}, k \in \mathbb{Z}_{+}$, where

$$
\mathcal{L}_{0}=\{[0,1)\} \quad \text { and } \quad \mathcal{L}_{k}=\left\{j+2^{-r k}[0,1): j=0,1,2, \ldots r^{k}-1\right\} .
$$

For the case considered in [8], our definition coincides with the definition given there. The easiest way to see this equivalence is to look directly at the proof of Theorem 2 in [8] (at least that was the easiest way for me).

Note also, that for homogeneous lattices the norms $\|f\|_{p}|I|^{-1 / p}$ on $D_{I}$ are all equivalent. This means that any (weakly or strongly) $(p, \varepsilon)$ mixing martingale transform is also $(p, \varepsilon, K)$ mixing (resp. weakly or strongly) with appropriate $K$. It also mean that any $(p, \varepsilon)$ mixing martingale transform is also $\left(r, \varepsilon^{\prime}\right)$ mixing with appropriate $\varepsilon^{\prime}$.

Recall that we defined the formal sum $b_{0}=\sum_{I \in \mathcal{L}} \Delta_{I} b$. Define also the formal sum

$$
\widetilde{b}_{0}:=\sum_{I \in \mathcal{L} \backslash \mathfrak{A}_{-\infty}^{0, \text { fin }}} \Delta_{I} b
$$

(note that $b_{0}=\widetilde{b}_{0}$ if $\mathfrak{A}_{-\infty}^{0, \text { fin }} \cap \mathcal{L}=\varnothing$ ).

Theorem 5.3. Let $p \in(1, \infty)$ and let $T$ be a strongly $(p, \varepsilon, K)$ mixing martingale transform, such that its blocks $T_{I}$ are uniformly bounded in $L^{p}{ }^{2}$

If the commutator $\left[T, M_{b}\right]$ is bounded in $L^{p}$, then $\widetilde{b}_{0} \in \mathcal{B M O}=\mathcal{B M O}_{2}$.

Moreover, for $p=2$ it is sufficient to assume that $T$ is a weakly $(2, \varepsilon, K)$ mixing martingale transform.

Finally, the norm $\left\|\widetilde{b}_{0}\right\|_{\mathcal{B M O}}$ can be estimated by a constant depending on $p$, $\left\|\left[M_{b}, T\right]\right\|_{L^{p} \rightarrow L^{p}}, \sup _{I \in \mathcal{L}}\left\|T_{I}\right\|$, and $\varepsilon$ and $K$ from Definition 5.2 .

Proposition 5.4. Let $p \in(1, \infty)$ and let $T$ be a (possibly unbounded) strongly $(p, \varepsilon)$ mixing martingale transform (weakly $(p, \varepsilon)$ mixing for $p=2)$.

If the commutator $\left[M_{b}, T\right]$ is bounded in $\mathcal{H}_{2}^{p}$, then for any interval $I \in \mathcal{L} \backslash \mathfrak{A}_{-\infty}^{0, \text { fin }}$, there holds the uniform estimate

$$
f_{I}\left(\sum_{J \in \mathcal{L}: J \subset I}\left|\Delta_{J} b\right|^{2}\right)^{p / 2} d x \leq C<\infty, \quad C^{1 / p}=C_{1}\left\|\left[M_{b}, T\right]\right\| / \varepsilon .
$$

where $C_{1}=C_{1}(p)$.

Proof of Proposition 5.4. The proof follows [8] directly. For an interval $I$, let $I^{\prime}$ be its parent, so $I \in \operatorname{child}\left(I^{\prime}\right)$. We know that $I$ is $(p, \varepsilon)$ non-degenerate. Let $h=h_{I^{\prime}} \in D_{I^{\prime}}$ be the function from Definition 5.2 such that $\|h\|_{p}=1,\left.h\right|_{I}=0$ and $\left\|\mathbf{1}_{I} T_{I^{\prime}} h\right\|_{p} \geq \varepsilon$. Note that $\|h\|_{p}=\|h\|_{\mathcal{H}_{2}^{p}}$.

\footnotetext{
${ }^{2}$ Note, that for $p \neq 2$ this condition is weaker than the boundedness of $T$ in $L^{p}$.
} 
Recall that the function $T h=T_{I^{\prime}} h$ is constant on $I$, and let $c$ be its value there. The inequality $\left\|\mathbf{1}_{I} T_{I^{\prime}} h\right\|_{p} \geq \varepsilon$ means that $|c| \geq \varepsilon|I|^{-1 / p}$.

We get that, on $I$,

$$
M_{b} T h=c b .
$$

On the other hand, $b h=0$ on $I$, so $\left.(T b h)\right|_{I}$ is a constant, so for $J \subset I$,

$$
\Delta_{J}\left(\left(M_{b} T-T M_{b}\right) h\right)=c \Delta_{J} b .
$$

The fact that $M_{b} T-T M_{b}$ is bounded in $\mathcal{H}_{2}^{p}$ implies that

$$
|c| \cdot\left\|\left(\sum_{J \in \mathcal{L} J \subset I}\left|\Delta_{J} b\right|^{2}\right)^{1 / 2}\right\|_{p} \leq C, \quad C=\left\|\left[M_{b}, T\right]\right\|,
$$

so taking into account that $|c| \geq \varepsilon|I|^{-1 / p}$ we get the conclusion of the proposition.

For $p=2$, we can assume that $T$ is weakly $(p, \varepsilon)$ mixing, because if $I$ is $(p, \varepsilon)$ non-degenerate for $T^{\prime}$, we can consider the adjoint of the commutator, to get the same conclusion. This would not work for $p \neq 2$, because in this case we get the estimate with the exponent $p^{\prime}$ instead of $p$.

5.2.1. Proof of Theorem 5.3. To prove the theorem we need to show that $\left\|\Delta_{I} b\right\|_{\infty}$ are uniformly bounded for all $I \in \mathcal{L} \backslash \mathfrak{A}_{-\infty}^{0, \text { fin }}$.

Consider an interval (let us call it $I^{\prime}$ ) belonging to $\mathcal{L} \backslash \mathfrak{A}_{-\infty}^{0, \text { fin }}$. Notice that inequality (5.2) implies that $\left\|\Delta_{I^{\prime}} b\right\|_{p} \leq C^{1 / p}\left|I^{\prime}\right|^{1 / p}<\infty$. Assume that $M:=\left\|\Delta_{I^{\prime}} b\right\|_{\infty}$ is attained on $I \in \operatorname{child}\left(I^{\prime}\right)$.

We can assume that $|I|<\left|I^{\prime}\right| / K$, because otherwise

$$
\left\|\mathbf{1}_{I} \Delta_{I^{\prime}} b\right\|_{\infty}^{p}=|I|^{-1}\left\|\mathbf{1}_{I} \Delta_{I^{\prime}} b\right\|_{p}^{p} \leq|I|^{-1}\left\|\Delta_{I^{\prime}} b\right\|_{p}^{p} \leq|I|^{-1} C\left|I^{\prime}\right| \leq K C .
$$

Define

$$
g=\mathbf{1}_{I}-\gamma \mathbf{1}_{I^{\prime} \backslash I},
$$

where the constant $\gamma$ is chosen so $\int_{\mathbb{X}} g d x=0$. Let $E \subset D_{I^{\prime}}$ be the annihilator of $g$ in $D_{I^{\prime}}$,

$$
E=\left\{f \in D_{I^{\prime}}: \int_{\mathbb{X}} g f d x=0\right\} .
$$

Note that $E$ consist of all functions $f \in D_{I^{\prime}}$ supported outside of $I$. Indeed, any such function annihilates $g$, and counting dimensions, we can conclude that these are all the functions in the annihilator.

This structure for $E$ implies that

$$
\int_{\mathbb{X}} f \bar{g} d x=0 \quad \forall f \in E
$$

so $E$ is the orthogonal complement of $g$ in $D_{I^{\prime}}$. Therefore, $D_{I^{\prime}}$ can be decomposed as the direct sum of $\operatorname{span}\{g\}$ and $E$.

Let $h=h_{I^{\prime}}$ be the function in Definition 5.2. We can decompose

$$
\left(\Delta_{I^{\prime}} b\right) T_{I^{\prime}} h=\alpha g+f+\mathbb{E}_{I^{\prime}}\left[\left(\Delta_{I^{\prime}} b\right)\left(T_{I^{\prime}} h\right)\right], \quad f \in E .
$$


By the assumption (iii) from Definition 5.2, $\left|T_{I^{\prime}} h\right| \geq \varepsilon|I|^{-1 / p}$ on $I$. Therefore, since $\left.f\right|_{I}=0$ and $\left.g\right|_{I}=1$, from (5.3) we get, by restricting to $I$ and comparing $L^{p}$ norms (divided by $|I|^{1 / p}$ ), that

$\varepsilon|I|^{-1 / p}\left\|\Delta_{I^{\prime}} b\right\|_{\infty} \leq|\alpha|+\left\|\mathbb{E}_{I^{\prime}}\left[\left(\Delta_{I^{\prime}} b\right)\left(T_{I^{\prime}} h\right)\right]\right\|_{\infty}$

$$
\leq|\alpha|+\left|I^{\prime}\right|^{-1}\left\|\Delta_{I^{\prime}} b\right\|_{p^{\prime}}\left\|T_{I^{\prime}}\right\|\|h\|_{p} \leq|\alpha|+C\left|I^{\prime}\right|^{-1}\left\|\Delta_{I^{\prime}} b\right\|_{p^{\prime}} .
$$

So, to estimate $\left\|\Delta_{I^{\prime}} b\right\|_{\infty}$ we need to estimate both terms on the right side of (5.4).

We get the bound on $|\alpha|$ from the boundedness of the commutator. Namely, since

$$
\left.b\right|_{I^{\prime}}=\mathbb{E}_{I^{\prime}} b+\Delta_{I^{\prime}} b+\sum_{J \in \mathcal{L}: J \varsubsetneqq I^{\prime}} \Delta_{J^{\prime}} b=: \mathbb{E}_{I^{\prime}} b+\Delta_{I^{\prime}} b+b^{I^{\prime}}
$$

and $b^{I^{\prime}} \perp D_{I^{\prime}}, b^{I^{\prime}} D_{I^{\prime}} \perp D_{I^{\prime}}$, we can write

$$
\begin{aligned}
& \left\langle M_{b} T h, g\right\rangle=\left\langle M_{b} T_{I^{\prime}} h, g\right\rangle=\left\langle\left(\Delta_{I^{\prime}} b\right) T_{I^{\prime}} h, g\right\rangle+\left\langle\left(\mathbb{E}_{I^{\prime}} b\right) T_{I^{\prime}} h, g\right\rangle, \\
& \left\langle T M_{b} h, g\right\rangle=\left\langle T_{I^{\prime}} M_{b} h, g\right\rangle=\left\langle T_{I^{\prime}}\left(\Delta_{I^{\prime}} b\right) h, g\right\rangle+\left\langle T_{I^{\prime}}\left(\mathbb{E}_{I^{\prime}} b\right) h, g\right\rangle .
\end{aligned}
$$

Here we slightly abuse notation by treating $T_{I^{\prime}}$ as an operator on all of $L^{p}$, i.e., as a martingale transform whose only nonzero block is $T_{I^{\prime}}$ (we need to do that because $\left(\Delta_{I^{\prime}}, b\right) h$ does not generally belongs to $\left.D_{I^{\prime}}\right)$. In this context $T_{I^{\prime}}\left(\Delta_{I^{\prime}}, b\right) h=$ $T_{I^{\prime}}\left[\left(\Delta_{I^{\prime}} b\right) h-\mathbb{E}_{I^{\prime}}\left(\left(\Delta_{I^{\prime}} b\right) h\right)\right]$, where $T_{I^{\prime}}$ on the right side can be treated as a block acting on $D_{I^{\prime}}$.

Using the fact that $\left(\mathbb{E}_{I^{\prime}}, b\right) T_{I^{\prime}} h=T_{I^{\prime}}\left(\mathbb{E}_{I^{\prime}}, b\right) h$ we conclude, abusing notation as above, that, for the commutator $\left[M_{b}, T\right]=M_{b} T-T M_{b}$,

$$
\left\langle\left[M_{b}, T\right] h, g\right\rangle=\left\langle\left(\Delta_{I^{\prime}} b\right) T_{I^{\prime}} h, g\right\rangle-\left\langle T_{I^{\prime}}\left(\Delta_{I^{\prime}} b\right) h, g\right\rangle .
$$

We get from (5.3) that

$$
\left|\left\langle\left(\Delta_{I^{\prime}} b\right) T_{I^{\prime}} h, g\right\rangle\right|=|\alpha| \cdot\|g\|_{2}^{2} \geq|\alpha| \cdot|I| .
$$

By Lemma 4.7, $\|g\|_{p^{\prime}} \leq 2^{1 / p^{\prime}}\left\|\mathbf{1}_{I}\right\|_{p^{\prime}}=2^{1 / p^{\prime}}|I|^{1 / p^{\prime}}$. Using this estimate and the assumption $\|h\|_{\infty} \leq K\left|I^{\prime}\right|^{-1 / p}$, we get

$$
\begin{aligned}
\left|\left\langle T_{I^{\prime}}\left(\Delta_{I^{\prime}} b\right) h, g\right\rangle\right| & \leq\left\|T_{I^{\prime}}\right\| \cdot\|h\|_{\infty}\left\|\Delta_{I^{\prime}} b\right\|_{p}\|g\|_{p^{\prime}} \\
& \leq C K\left|I^{\prime}\right|^{-1 / p} C\left|I^{\prime}\right|^{1 / p} 2^{1 / p}|I|^{1 / p^{\prime}} \leq C|I|^{1 / p^{\prime}} .
\end{aligned}
$$

Using the above estimate together with the estimate

$$
\left|\left\langle\left[M_{b}, T\right] h, g\right\rangle\right| \leq C\|h\|_{p}\|g\|_{p^{\prime}} \leq C \cdot 1 \cdot|I|^{1 / p^{\prime}}
$$

we get from (5.5) and (5.6) that

$$
\begin{aligned}
|\alpha| \cdot|I| & \leq\left|\left\langle\left(\Delta_{I^{\prime}} b\right) T_{I^{\prime}}, h, g\right\rangle\right| \leq\left|\left\langle\left[M_{b}, T\right] h, g\right\rangle\right|+\left|\left\langle T_{I^{\prime}}\left(\Delta_{I^{\prime}} b\right) h, g\right\rangle\right| \\
& \leq C|I|^{1 / p^{\prime}}+C|I|^{1 / p^{\prime}}=C|I|^{1 / p^{\prime}},
\end{aligned}
$$

SO

$$
|\alpha| \leq C|I|^{-1 / p} .
$$


Combining the last inequality with (5.4) we get

$$
\begin{aligned}
\left\|\Delta_{I^{\prime}} b\right\|_{\infty} & \leq \frac{|I|^{1 / p}}{\varepsilon}|\alpha|+C \frac{|I|^{1 / p}}{\varepsilon}\left\|\Delta_{I^{\prime}} b\right\|_{p^{\prime}}\left|I^{\prime}\right|^{-1} \\
& \leq C+C|I|^{1 / p}\left|I^{\prime}\right|^{-1}\left\|\Delta_{I^{\prime}} b\right\|_{p^{\prime}} .
\end{aligned}
$$

If $p^{\prime} \leq p$, the Hölder inequality implies that

$$
\left|I^{\prime}\right|^{-1 / p^{\prime}}\left\|\Delta_{I^{\prime}} b\right\|_{p^{\prime}} \leq\left|I^{\prime}\right|^{-1 / p}\left\|\Delta_{I^{\prime}} b\right\|_{p} \leq C,
$$

so

$$
\left\|\Delta_{I^{\prime}} b\right\|_{\infty} \leq C+C|I|^{1 / p}\left|I^{\prime}\right|^{-1 / p} \leq C^{\prime} .
$$

If $p^{\prime}>p$, Lemma 5.5 below implies that

$$
\left\|\Delta_{I^{\prime}} b\right\|_{p^{\prime}} \leq\left\|\Delta_{I^{\prime}} b\right\|_{p}^{p / p^{\prime}}\left\|\Delta_{I^{\prime}} b\right\|_{\infty}^{1-p / p^{\prime}}
$$

and we get, from (5.7),

$$
\begin{aligned}
\left\|\Delta_{I^{\prime}} b\right\|_{\infty} & \leq C+C|I|^{1 / p}\left|I^{\prime}\right|^{-1}\left\|\Delta_{I^{\prime}} b\right\|_{p}^{p / p^{\prime}}\left\|\Delta_{I^{\prime}} b\right\|_{\infty}^{1-p / p^{\prime}} \\
& \leq C+C|I|^{1 / p}\left|I^{\prime}\right|^{-1 / p}\left\|\Delta_{I^{\prime}} b\right\|_{\infty}^{1-p / p^{\prime}}
\end{aligned}
$$

the last inequality being true because

$$
\left|I^{\prime}\right|^{-1 / p^{\prime}}\left\|\Delta_{I^{\prime}} b\right\|_{p}^{p / p^{\prime}}=\left(\left|I^{\prime}\right|^{-1 / p}\left\|\Delta_{I^{\prime}} b\right\|_{p}\right)^{p / p^{\prime}} \leq C^{p / p^{\prime}} \leq C^{\prime} .
$$

Since $|I| \leq\left|I^{\prime}\right|,(5.8)$ implies

$$
\left\|\Delta_{I^{\prime}} b\right\|_{\infty} \leq C+C\left\|\Delta_{I^{\prime}} b\right\|_{\infty}^{1-p / p^{\prime}},
$$

which gives us a bound $\left\|\Delta_{I^{\prime}} b\right\|_{\infty} \leq C^{\prime}$.

Lemma 5.5. Let $f$ be a bounded measurable function on a measure space $\mathbb{X}$. Then for any $q>p$

$$
\|f\|_{q} \leq\|f\|_{p}^{p / q}\|f\|_{\infty}^{1-p / q}
$$

Proof.

$$
\|f\|_{q}^{q}=\int_{\mathbb{X}}|f|^{q} d \mu=\int_{\mathbb{X}}|f|^{p}|f|^{q-p} d \mu \leq\|f\|_{\infty}^{q-p} \int_{\mathbb{X}}|f|^{p} d \mu=\|f\|_{\infty}^{q-p}\|f\|_{p}^{p},
$$

and raising this inequality to the power $1 / q$ we get the conclusion of the lemma.

\subsection{Relaxing the sufficient condition}

If $\mathcal{L} \cap \mathfrak{A}_{-\infty}^{0, \text { fin }}=\varnothing$, we have $b_{0}=\widetilde{b}_{0}$, so $b_{0} \in \mathcal{B M O}$ is a necessary and sufficient condition for the boundedness of the commutator $\left[M_{b}, T\right]$ (provided that $T$ satisfies the assumptions of Theorem 5.3). 
If $\mathcal{L} \cap \mathfrak{A}_{-\infty}^{0, \text { fin }} \neq \varnothing$ there is a gap between the necessary and sufficient conditions. Notice, that the situation $\mathcal{L} \cap \mathfrak{A}_{-\infty}^{0, \text { fin }} \neq \varnothing$ is not an exotic one. For example, it happens in the classical martingale situation, which in our notation means that $\mathcal{L}_{k}=\mathcal{L}_{0}=\mathbb{X}$ for all $k<0$ and $|\mathbb{X}|=1$.

To bridge the gap between the necessary and sufficient conditions in the case $\mathcal{L} \cap \mathfrak{A}_{-\infty}^{0, \text { fin }} \neq \varnothing$, we can relax the sufficient conditions in Proposition 5.1.

Proposition 5.6. Let $b$ be a locally integrable function, and let $T$ be a martingale transform bounded in $L^{p}$. Assume that

(i) $\widetilde{b}_{0} \in \mathcal{B M O}$, where $\widetilde{b}_{0}$ is defined by (5.1);

(ii) for any $I \in \mathcal{L} \cap \mathfrak{A}_{-\infty}^{0, \text { fin }}$,

$$
\left\|T_{I} \Delta_{I} b\right\|_{p} \leq C_{1}\left\|\mathbf{1}_{I}\right\|_{p}=C_{1}|I|^{1 / p}, \quad\left\|T_{I}^{*} \Delta_{I} b\right\|_{p^{\prime}} \leq C_{1}\left\|\mathbf{1}_{I}\right\|_{p^{\prime}}=C_{1}|I|^{1 / p^{\prime}} ;
$$

(iii) for any $I \in \mathcal{L} \cap \mathfrak{A}_{-\infty}^{0, \text { fin }}$,

$$
\left\|\left[\left(\Lambda_{b}^{1}\right)_{I}, T_{I}\right]\right\|_{L^{p \rightarrow L^{p}}} \leq C_{2}<\infty
$$

here $\left(\Lambda_{b}^{1}\right)_{I}$ is the restriction of $\Lambda_{b}^{1}$ to $D_{I}$.

Then the commutator $\left[M_{b}, T\right]$ is bounded in $L^{p}$, and

$$
\left\|\left[M_{b}, T\right]\right\|_{L^{p \rightarrow L^{p}}} \leq C\left(\|T\|_{L^{p \rightarrow L^{p}}}\left\|\widetilde{b}_{0}\right\|_{\mathcal{B M O}}+C_{1}+C_{2}\right),
$$

where $C=C(p)$, and $C_{1}$ and $C_{2}$ are the constants from (ii) and (iii).

The proof of the theorem is obvious, since for any $I \in \mathcal{L} \cap \mathfrak{A}_{-\infty}^{0, \text { fin }}$ conditions (ii) and (iii) are necessary and sufficient for the boundedness of the commutator $\left[M_{b_{0}-\widetilde{b}_{0}}, T\right]$ in $L^{p}$. The necessity here is quite easy: condition (ii) is obtained by testing the commutator $\left[M_{b_{0}-\widetilde{b}_{0}}, T\right]$ and its adjoint on the function $\mathbf{1}_{I}$. To get condition (iii) one needs to restrict everything to the subspace $D_{I}$.

Remark 5.7. As follows from the above discussion, if $T$ satisfies the assumptions of Theorem 5.3, then conditions (i)-(iii) of Proposition 5.6 are necessary and sufficient for the boundedness of the commutator $\left[M_{b}, T\right]$ in $L^{p}$.

\subsection{Some examples and counterexamples}

In this subsection we present examples which will show us that:

(i) Boundedness of the commutator $\left[M_{b}, T\right]$ does not imply any bounds on $\Delta_{I} b$ for $I \in \mathcal{L} \cap \mathfrak{A}_{-\infty}^{0, \text { fin }}$.

(ii) If the martingale transform $T$ is only strongly $(p, \varepsilon)$ mixing (not strongly $(p, \varepsilon, K)$ mixing), then the boundedness of the commutator $\left[M_{b}, T\right]$ does not imply any bounds on $\left\|\Delta_{I} b\right\|_{\infty}, I \in \mathcal{L}$. This means that the new condition (iv) in Definition 5.2 is essential and cannot be skipped.

The main building block of our construction will be as follows. Let an interval $I$ be divided into two subintervals $I^{1,2},\left|I^{1}\right| /\left|I^{2}\right|=\delta>0$. Divide $I^{1}$ into four equal intervals $I_{k}, 1 \leq k \leq 4$ and $I^{2}$ into four equal intervals $I_{k}, 5 \leq k \leq 8$. 
The intervals $I_{k}$ will be the children of $I$. Define the "Haar functions" $h^{k}=$ $h_{I}^{k} \in D_{I}$ by

$$
h^{k}:=\mathbf{1}_{I_{2 k}}-\mathbf{1}_{I_{2 k-1}}, \quad 1 \leq k \leq 4 .
$$

Note, that the functions $h^{k}$ do not span the martingale difference subspace $D_{I}$. Define also a "Haar function" $h=h_{I} \in D_{I}$ by $h=\mathbf{1}_{I^{1}}-\delta \mathbf{1}_{I^{2}}$.

On $D_{I}$, define a block $T_{I}$ by

$$
\begin{array}{ll}
T_{I} h^{1}=h^{2}, & T_{I} h^{2}=h^{1}, \\
T_{I} h^{3}=h^{4}, & T_{I} h^{4}=h^{3},\left.\quad T_{I}\right|_{\operatorname{span}\left\{h^{k}: 1 \leq k \leq 4\right\}^{\perp}}=0 .
\end{array}
$$

If $\Delta_{I} b=\alpha h_{I}$, then the block $\left(\Lambda_{b}^{1}\right)_{I}$ of $\Lambda_{b}^{1}$ commutes with $T_{I}$. This together with the fact that $T_{I} h_{I}=0$ implies that if $I \in \mathcal{L} \cap \mathfrak{A}_{-\infty}^{0 \text {,fin }}$ and the block $T_{I}$ of a martingale transform $T$ is as described above, then multiplication operator $M_{h_{I}}$ commutes with $T$.

So, if we add to $b$ any multiple of $h_{I}$, we will not be able to detect it by looking at the commutator $\left[M_{b}, T\right]$, which gives a example of the statement (i) above.

To give an example of statement (ii), take a finite interval $I_{0}=: \mathbb{X}$, divide it into 8 subintervals, as was described above (with $\delta=\delta_{1}$ ) to get the "children" of $I_{0}$, then divide each child into 8 parts, and so on. We assume that in each step we take $\delta=\delta_{n}, \delta_{n} \rightarrow 0$ as $n \rightarrow \infty$. This will be our lattice $\mathcal{L}$.

Let $T$ be a martingale transform on $\mathcal{L}$, where each block $T_{I}$ is as described above. Notice, that $T$ is strongly $(p, \varepsilon)$ mixing (but not strongly $(p, \varepsilon, K)$ mixing). Notice also, that $T$ is clearly bounded in $L^{2}$.

Take an interval $I \in \mathcal{L} \backslash \mathfrak{A}_{-\infty}^{0, \text { fin }}=\mathcal{L} \backslash\left\{I_{0}\right\}$.

Take $p=2$ and define $\widetilde{h}=\widetilde{h}_{I}=\delta^{-1 / 2} h$, where $h=h_{I}$ is the "Haar function" defined above, $h=\mathbf{1}_{I^{1}}-\delta \mathbf{1}_{I^{2}}$.

By Lemma 4.7, $\|\widetilde{h}\|_{2} \leq 2^{1 / 2}|I|^{1 / 2}$. On the other hand, $\|\tilde{h}\|_{\infty}=\delta^{-1 / 2}$, so we can pick $I$ such that $\|\widetilde{h}\|_{\infty}$ is as large as we want.

Note that for $b=\widetilde{h}$, the martingale transform $T$ commutes with $\Lambda_{b}^{1}$ (and so with $\Lambda^{b}$ ), so it is easy to check that the paraproducts $\pi_{b}$ and $\pi_{b}^{*}$, and so also the commutator $\left[M_{b}, T\right]$, are bounded. However, as we discussed above, $\|b\|_{\infty}=\delta^{-1 / 2}$.

So, if we consider a collection $\mathcal{C}$ of disjoint intervals in $\mathcal{L} \backslash \mathfrak{A}_{-\infty}^{0, \text { fin }}$ with $\delta \rightarrow 0$, and define

$$
b=\sum_{I \in \mathcal{C}} \widetilde{h}_{I}
$$

then the commutator $\left[M_{b}, T\right]$ is bounded. This can be seen, for example, by noticing that $\Lambda_{b}^{1}$ commutes with $T$ (one needs to treat each block separately, which reduces it to the case $b=\widetilde{h}_{I}$ ), and the paraproducts $\pi_{b}$ and $\pi_{b}^{*}$ are "direct sums" of the paraproducts with $b=\widetilde{h}_{I}$, treated above.

So we constructed an example of $b$ and a strongly $(p, \varepsilon)$ mixing martingale transform $T$ such that the commutator $\left[M_{b}, T\right]$ is bounded in $L^{2}$, but $\sup _{I \in \mathcal{L}}\left\|\Delta_{I}\right\|_{\infty}=\infty$.

An easy modification allows also to get an example for $L^{p}$. 


\section{References}

[1] Blasco, O. and Pott, S.: Dyadic BMO on the bidisk. Rev. Mat. Iberoamericana 21 (2005), no. 2, 483-510.

[2] Burkholder, D. L.: Explorations in martingale theory and its applications. In École d'Été de Probabilités de Saint-Flour XIX, 1989, 1 -66. Lecture Notes in Math 1464, Springer, Berlin, 1991.

[3] Davis, B.: Hardy spaces and rearrangements. Trans. Amer. Math. Soc. 261 (1980), no. 1, 211-233.

[4] Davis, B.: On the integrability of the martingale square function. Israel J. Math. 8 (1970), 187-190.

[5] Fefferman, C. and Stein E. M.: Some maximal inequalities. Amer. J. Math. 93 (1971), 107-115.

[6] Frazier, M. And Jawerth, B.: A discrete transform and decompositions of distribution spaces. J. Funct. Anal. 93 (1990), no. 1, 34-170.

[7] Garsia, A. M.: Martingale inequalities: Seminar notes on recent progress. Mathematics Lecture Notes Series, Reading, Mass.-London-Amsterdam, 1973.

[8] JAnson, S.: BMO and commutators of martingale transforms. Ann. Inst. Fourier (Grenoble) 31 (1981), no. 1, viii, 265-270.

[9] Nazarov, F. And Treil, S.: The hunt for a Bellman function: applications to estimates for singular integral operators and to other classical problems of harmonic analysis. Algebra i Analiz. 8 (1996), no. 5, 32-162

[10] Nazarov, F., Treil, S. And Volberg, A.: The Tb-theorem on non-homogeneous spaces. Acta Math. 190 (2003), no. 2, 151-239.

[11] Stein, E. M.: Harmonic analysis: real-variable methods, orthogonality, and oscillatory integrals. Princeton Mathematical Series 43, Monographs in Harmonic Analysis III, Princeton University Press, Princeton, NJ, 1993.

[12] Tolsa, X.: BMO, $H^{1}$, and Calderón-Zygmund operators for non doubling measures. Math. Ann. 319 (2001), no. 1, 89-149.

[13] Tolsa, X.: Painlevé's problem and the semiadditivity of analytic capacity. Acta Math. 190 (2003), no. 1, 105-149.

[14] Weisz, F.: Martingale Hardy spaces and their applications in Fourier analysis. Lecture Notes in Mathematics 1568, Springer-Verlag, Berlin, 1994.

[15] WeIsz, F.: Martingale operators and Hardy spaces generated by them. Studia Math. 114 (1995), no. 1, 39-70.

Received January 3, 2012; revised January 11, 2013.

Sergei Treil: Department of Mathematics, Brown University, 151 Thayer Str./Box 1917, Providence, RI 02912, USA.

E-mail: treil@math.brown.edu

This material is based on work supported by the National Science Foundation under the grant DMS-0800876. Any opinions, findings and conclusions or recommendations expressed in this material are those of the author and do not necessarily reflect the views of the National Science Foundation. 